\title{
REVIEW OF PHYSICS RESULTS FROM THE TEVATRON: SEARCHES FOR NEW PARTICLES AND INTERACTIONS
}

\author{
DAVID TOBACK \\ Mitchell Institute for Fundamental Physics and Astronomy \\ Department of Physics and Astronomy, Texas A\&M University \\ College Station, TX 77843-4242, USA \\ toback@tamu.edu \\ LIDIJA ŽIVKOVIĆ \\ Laboratory for High Energy Physics, Institute of Physics Belgrade \\ Pregrevica 118, 11080 Zemun, Serbia \\ lidiaz@fnal.gov
}

Received Day Month Year

Revised Day Month Year

\begin{abstract}
We present a summary of results for searches for new particles and interactions at the Fermilab Tevatron collider by the CDF and the D0 experiments. These include results from Run I as well as Run II for the time period up to July 2014. We focus on searches for supersymmetry, as well as other models of new physics such as new fermions and bosons, various models of excited fermions, leptoquarks, technicolor, hidden-valley model particles, long-lived particles, extra dimensions, dark matter particles, and signaturebased searches.

Keywords: Tevatron, CDF, D0, supersymmetry, charged massive stable particles, heavier vector bosons and fermions, excited fermions, leptoquarks, technicolor, hidden-valley models, long-lived particles, extra dimensions, dark matter

PACS numbers: 14.65.Jk, 14.70.Pw, 14.70.Kv, 14.80.Nb, 14.80.Sv, 14.80.Tt, 14.80.Ly, 14.80.Rt, 13.85.Rm
\end{abstract}


Contents

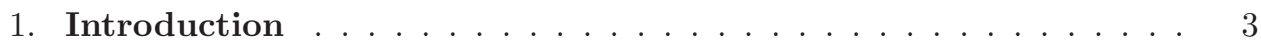

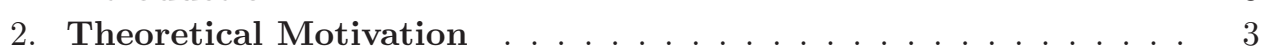

2.1. Supersummetry . . . . . . . . . . . . . . . . . 4

2.2. Resonances ...................... 5

2.3. Hidden-valley models, CHAMPS and long-lived particles . 6

2.4. Extra dimensions and dark matter . . . . . . . . . . . 6

2.5. Siqnature-based searches and model-independent searches . 7

3. Run I Results . . . . . . . . . . . . . . . . . . . . . 8

3.1. The eer $\gamma E_{T}$ candidate event and its influence . . . . . . . . 8

3.2. Follow up on the leptoquark hints from DESY . . . . . . . 9

3.3. Siqnature-based searches and SLEUTH . . . . . . . . . . . 9

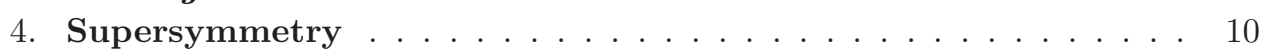

4.1. mSUGRA/Heavy LSP models . . . . . . . . . . . . . . . 11

4.1.1. Light flavor squarks and gluinos . . . . . . . . . . . . 11

4.1.2. Bottom and top squarks . . . . . . . . . . . . . . . 13

4.1.3. Gauqinos ...................... 14

4.2. Gauge-mediated supersymmetry breaking (GMSB) models . 15

4.3. R-parity violation . . . . . . . . . . . . . . . . . . 17

5. Other BSM Searches . . . . . . . . . . . . . . . . . . . . 17

5.1. Resonances . . . . . . . . . . . . . . . 17

5.1.1. New fermions and bosons . . . . . . . . . . . . . . 17

5.1.2. Excited fermions . . . . . . . . . . . . . . . . . . 23

5.1.3. Leptoquarks . . . . . . . . . . . . . . . . . . . . . . 23

5.1.4. Technicolon . . . . . . . . . . . . . . . . 24

5.1.5. Other resonance searches . . . . . . . . . . . . 24

5.2. Hidden-valley models, CHAMPS and long-lived particles . 25

5.2.1. Hidden-valley/dark photons . . . . . . . . . . . . . . . 26

5.2.2. Charged massive stable particles _. . . . . . . . . . . 27

5.2.3. Monopoles, stopped qluinos and quirks . . . . . . . . . . 28

5.3. Extra dimensions and dark matter . . . . . . . . . . . . . . 29

5.4. Signature-based searches and model-independent searches . 32

6. Summary and Conclusions . . . . . . . . . . . . . . . . 33 


\section{Introduction}

The standard model (SM) of particle physics has had great success describing the known particles, their properties and the interactions between them, up to energies of about $1 \mathrm{TeV} !^{1}$ The recent discovery of the Higgs boson ${ }^{2}[3]$ completed the model, but there are still many unanswered questions as well as many unexplained phenomena that remain.

In this review we present a summary of results for searches for new particles and interactions at the Fermilab Tevatron collider. These include results from Run I as well as Run II which produced about $10 \mathrm{fb}^{-1}$ of $p \bar{p}$ collisions at $\sqrt{s}=1.96 \mathrm{TeV}$ recorded by each experiment. We focus on searches for supersymmetry (SUSY) , new fermions and bosons, excited fermions, leptoquarks, technicolor particles, hidden-valley model particles, long-lived particles, extra dimensions, dark matter, and signature-based searches. While we will not discuss the full set of searches, the references contain a fairly complete set of results. Many other searches for new particles and interactions that are not presented here (e.g. non-SM Higgs boson searches, $B_{s} \rightarrow \mu \mu$ ) are presented in the different chapters of this review.

We begin with a quick overview of some of the theoretical motivations that influenced the set of searches that were ultimately done by the experiments. In section 3 we provide a historical review of some of the Run I results that had a large impact on the world-wide searches, including the ee $\gamma \gamma \mathbb{E}_{T}$ candidate event, follow-ups on the leptoquark hints from the DESY ep collider (HERA), signaturebased searches like SLEUTH and other searches that kept the Fermilab Tevatron collider experiments at the frontier. In section 4 we discuss the Run II SUSY results and in section 5 we discuss the various other beyond the standard model (BSM) searches results from Run II. In section 6 we conclude.

\section{Theoretical Motivation}

There are many reasons to search for new particles and interactions beyond the SM, and different theoretical viewpoints can guide the ways in which we search. On one end of the search strategy spectrum is the fact that we have many compelling and well specified models of BSM physics which predict new particles and how to look for them. On the opposite end of the spectrum, it is possible that we have not guessed the new physics, but the Tevatron collider has the ability to produce these new particles. Searching must also be done thoughtfully and carefully in more modelindependent ways to be ready for surprises. In this section we provide an overview of both types of motivations, with others in between the two extremes, with an eye towards searches. We will point the reader to more details on theoretical issues as they are discussed extensively in the literature; our references here are not intended to be complete but rather a guide for the reader to get started. Phenomenological

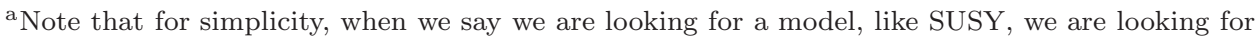
evidence of new particles and/or interactions. 
issues, like production mechanisms, decay products, final states and relevant models parameters are discussed in sections 4 and 5 .

\subsection{Supersymmetry}

The motivations for SUSY are well known and documented $\frac{4}{47}$ and include its ability to potentially solve hierarchy problem for the Higgs boson mass, provide a dark matter candidate, and satisfy consistency requirements of modern models of string theory. Inherent in the theory is that for every fermion observed in the SM there is a supersymmetric boson partner that has not yet been observed; the same is true for the known bosons, including the observed Higgs boson, and the hypothetical graviton (the particle mediator of gravity). The non-observation of low-mass sparticles with equal masses to their SM counterparts has focused efforts on SUSY models with broken symmetry.

Since there are many new particles to be searched for, and a 128 free parameters in the most general models, other "clues" and possible tie-ins have been used by model builders to focus on weak-scale SUSY $\frac{8}{8}$ The hallmark of these SUSY models is their ability to provide a dark matter candidate, and not contradict other observations. ${ }^{[9}$ Since experimental results from the proton and electron lifetime measurements imply conservation of baryon number and lepton number, it is not unreasonable that SUSY has an additional symmetry, known as $R$-parity 6 . If $R$-parity is conserved this has the consequence that the lightest SUSY particle (LSP) must be stable, potentially making it a dark matter candidate. We note for now that for many SUSY models the LSP couple to normal matter with a tiny strength and when produced in a collision, it would leave the detector without a trace, yielding significant missing transverse energy, $\mathbb{E}_{T}$, giving a signature for SUSY that is searched for in many models.

With this in mind we quickly mention the models focused on at the Fermilab Tevatron collider which are typically selected for simplicity and general features. These include: (i) gravity-mediated SUSY breaking (minimal SuperGravity or mSUGRA) type models, where the lightest neutralino is the LSP, has a mass at the electroweak scale and becomes a natural cold-dark matter candidate (discussed in section 4.1), (ii) gauge-mediated SUSY breaking models (GMSB) which have a $\sim \mathrm{keV}$ mass gravitino as the LSP, and often have a photon and $E_{T}$ in the final state (discussed in section 4.2), and (iii) $R$-parity violating (RPV) searches which release the desire to solve the dark matter problem with SUSY, but must be considered in the most general SUSY frameworks (discussed in 4.3). Other models that contain SUSY, like hidden-valley models, models that include charged massive stable particles (CHAMPS), etc, are discussed more in section 2.3 for their theory, and section 5.2 for results.

${ }^{\mathrm{b}} R=(-1)^{3 B+L+2 S}$, where $B$ is baryon number, $S$ is spin and $L$ is lepton number. 


\subsection{Resonances: new fermions and bosons, excited fermions, leptoquarks, technicolor and other new particles}

There are many other models of new physics which also predict new particles. For example, models which extend the gauge structure of the SM generically predict new gauge bosons and possibly new scalars and fermions. These new patterns may be produced on- (or nearly on-) shell and decay into SM particles yielding a telltale bump in an invariant mass spectrum. We search for resonances in a general way, but optimize and report our sensitivity to a small number of specific model types. These include models that contain new fermions and/or bosons, excited fermions, leptoquarks, as well as particles from technicolor and other models. We next describe some of the models that garnered the most attention during Runs I and II. Note that typically the mass of any new particle is the most relevant parameter of the theory, from a phenomenological standpoint, but when there are other parameters of importance we note them in section 5

New fermions are predicted in many BSM models. While there are significant experimental constraints from LEP, 10 and many models of extra dimensions would give an unobserved large enhancement of the Higgs boson cross section if there are extra fermions, $\frac{11}{11}$ there is currently no compelling theoretical reason for there to be three and only three fermion generations in the SM. Thus, it becomes natural to look for fourth generation chiral quarks and leptons, $\frac{12}{12}$ and vector-like quarks 13 (which have right-handed and left-handed components that transform in the same way under $S U(3) \times S U(2) \times U(1))$.

Similarly, additional bosons are predicted in many new models. For example, new gauge bosons are predicted in the minimal extensions of the SM that restore left-right symmetry $14 \sqrt[16]{16}$ with the gauge group $S U(2)_{L} \times S U(2)_{R}$. In these theories additional $W$ and $Z$ bosons, usually denoted as $W^{\prime}$ and $Z^{\prime}$, will couple to the righthanded fermions with weak coupling strength. In addition, grand unified theories and other theories also predict the existence of new heavy bosons, where often the gauge group can be broken to the SM gauge group or have additional $U(1)$ 's which could yield multiple $Z^{\prime}$ bosons. For a review of neutral heavy bosons, see Ref. 17 .

The simple organization of the SM particles into a table that resembles the periodic table of elements is suggestive that the known "fundamental" particles may actually be composite or otherwise have substructure ${ }^{[18}$ This idea is inherent in string theory 19 or models of technicolor (more below). If the known particles were composite, excited versions of each SM particle could be produced (like the excited states of atoms or hadrons); signatures of excited leptons could involve the production and decay $\ell^{*} \rightarrow \ell \gamma$, or, for excited quarks, $q^{*} \rightarrow q \gamma$.

Many grand unified theory models have unification of the quarks and leptons at the highest energies, suggesting the possibility of leptoquarks $(L Q)$ in nature 20 These new particles are color-triplet bosons, carry both quark and lepton quantum numbers, and have fractional electric charge, but their spin can be 0 (scalar $L Q$ ) or 1 (vector $L Q$ ). They could produce resonant signatures in the $\ell q$ or $\nu q$ final state. 
Theories of strong dynamics, such as technicolor,21 24 predict a host of new particles known as technifermions. In many ways this model posits that there are no fundamental bosons, and that the vector bosons and the Higgs boson are composite objects made of technifermions. An advantage of this model is that it removed the need of the only fundamental scalar in the SM, the Higgs boson and/or explain why it had not been observed in Run I or at LEP. With the discovery of the Higgs boson with SM properties, these models have fallen out of favor. One of the up sides of these models, is that they did provide natural search strategies for a number of resonances which were followed.

Another resonance search is for the production of light axigluons which can produce an anomalous top-quark forward-backward asymmetry $25,27 A_{f}$. . Alternative axigluon decay modes include low mass, strongly interacting particles which will further decay to pairs of jets, yielding resonances in the four-jet final states. These final states are also predicted by various theories where no intermediate resonance is necessary.

The searches for resonances from new fermions and bosons are presented in section 5.1.1 Similarly, searches for excited fermions, leptoquarks and technicolor are presented in sections 5.1.25.1.4. Other searches for other resonances, such as $Z \rightarrow \gamma \gamma$ and the $W+$ dijet search from CDF in Run II,, 29 are presented in section 5.1 .5 .

\subsection{Hidden-valley models, CHAMPS and other long-lived particles}

During Run II hidden-valley (HV) models were constructed that predict a new, confining gauge group that is weakly coupled to the standard model, leading to the production of new particles. These low mass particles could help explain potential hints in astrophysical and dark matter searches. These models are often incorporated into SUSY models with sparticles known as "dark particles", and a hallmark of their production is the decay of long-lived particles with unusual signatures in the detectors $: 30+32$

Other models of new long-lived particles include charged massive particles (CHAMPS) which are predicted in many models of new physics, especially in SUSY models ${ }^{33}$ Dirac monopoles have also been predicted for many years in GUT models, 9 and models that symmetrize electromagnetism. Finally, a new class of models predict new particles known as quirks which arise when there is a new, unbroken $S U(N)$ gauge group added beyond the $\mathrm{SM} \cdot 34$

\subsection{Extra dimensions and dark matter}

Many versions of string theory posit (and in most cases require) the existence of other dimensions in addition to our three spatial + one time dimensions. There are

${ }^{\mathrm{c}}$ More details can be found in the top quark chapter of this review 
a number of different types of models which have received the most attention. The first are large extra dimension (LED) model ${ }^{35}$ which postulate the existence of two or more extra dimensions in which only gravity can propagate. The weakness of gravity can thus be explained by a propagation through higher-dimension space. In universal extra dimensions (UED) model $s^{36}$ extra spatial dimensions are accessible to all SM fields. Consequently, the difference between UED and LED is that the spatial dimensions in UED are compactified resulting in KK excitations, which are "towers" of the SM fields. A third model is warped extra dimensions ${ }^{37}$ in which the existence of the fifth dimension, with a warped spacetime metric, is bounded by two three-dimensional branes. The SM fields and gravity are on different branes with a small overlap, causing gravity to appear weak at the $\mathrm{TeV}$ scale.

Dark matter has been inferred from the dynamics of galaxies and clusters of galaxies for decades, and the evidence that it is due to a new kind of elementary particle has steadily mounted 38 There have been many models of dark matter put forward by the community, but from the perspective of searches at the Fermilab Tevatron collider there have been just a few types that can be searched for: (i) production and decay of SUSY particles into dark matter particles (typically the LSP), (ii) axions, $\frac{40}{, 0}$ and (iii) theory-independent models of production. While SUSY has already been mentioned, we quickly note that axion production is out of reach of colliders. Recently, more model-independent searches have been done with few assumptions about the new physics and focus on the direct production of dark matter.

\subsection{Signature-based searches and model-independent searches}

While model-based searches have always been favored by the theory community, signature-based searches became an important part of the search program as a more model-independent way to search for physics beyond the SM. Perhaps by observing the unexpected we could find explanations of various unexplained phenomena (dark matter, electroweak symmetry breaking etc.). Indeed, looking at history, many of the major discoveries in particle physics have been made in unexpected ways (the muon is a prime example). For these reasons, powerful and systematic ways of searching for new physics were developed to help ensure that the unexpected was not missed. New methodologies focused on the idea of just looking at the final state particles to see if there was any indication of an unexpected resonance, or anomalous number of events or kinematic distribution when considering combinations of high transverse momentum $\left(p_{T}\right)$ particles. This method of searching with a set of final state particles is known as a "signature-based" search; because it is not looking for any particular new model, it is known as model-independent. Many different variations on these themes were created and executed, starting strongly in Run I (especially after the unexpected observation of an event with two electrons, two photons and $E_{T}$ ) and continuing throughout the search program. 


\section{Run I Results}

The Run I dataset consists of $\sim 100 \mathrm{pb}^{-1}$ of collision data at $\sqrt{s}=1.8 \mathrm{TeV}$. Most searches 1128 focused on the simplest resonance models, mSUGRA searches and RPV models. With the world's then highest energy collisions, these were the cutting edge, bracketed on each side by complementary searches from LEP. We learned that the SM worked up to a higher energy scale since there was no evidence for new physics other than the discovery of the top quark. However, there were some results that were exciting enough to have significant impact on the field. We mention three before proceeding to the Run II results.

\subsection{The ee $\gamma \gamma \ddot{H}_{T}$ candidate event and its influence}

During Run I, the CDF experiment observed a very unusual event which created significant interest 62 This event had two high energy electron candidates, two high energy photons and large $E_{T}$ (see Fig. 1(a)). Of particular note was that the $E_{T}$ was $55 \mathrm{GeV}$ and that the event could not be readily explained as a $W \rightarrow e \nu, Z \rightarrow e e$ or radiative versions of any combination of the above. There were no searches for this type of event at the time, and while the large $E_{T}$ was suggestive of SUSY, there were no models that were in favor that had photons in the final state.

(a)

\section{e err $\mathbb{Z}_{\mathrm{T}} \mathrm{C}$ andid ate Event}

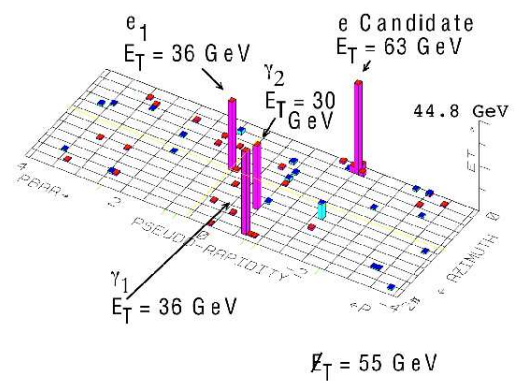

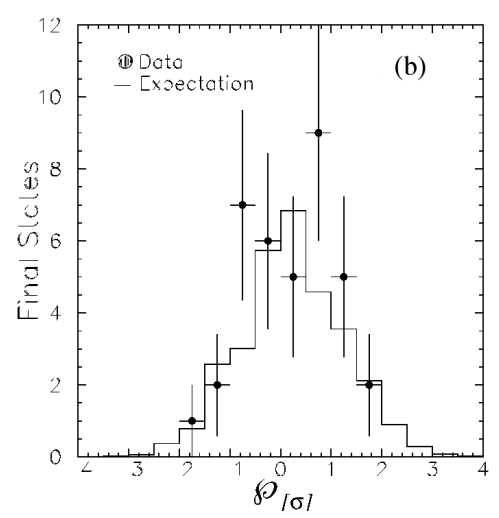

Fig. 1. (color online) (a) An event display of the CDF ee $\gamma \gamma \mathbb{E}_{T}$ candidate event observed in Run I. (b) The significance $\mathcal{P}$ of the excess, in units of standard deviations, obtained using SLEUTH at the D0 experiment from Run I.

While a detailed description of the set of models which were proposed to explain it is beyond the scope of this review, a long lasting impact was the rise of interest in GMSB SUSY. Example production and decay chains include $\tilde{e} \tilde{e} \rightarrow\left(e \tilde{\chi}_{1}^{0}\right)\left(e \tilde{\chi}_{1}^{0}\right) \rightarrow e(\gamma \tilde{G}) e(\gamma \tilde{G}) \rightarrow e e \gamma \gamma E_{T}$, or similar ones from chargino pair production and decay with virtual $W$ bosons ${ }^{8}$ GMSB has been a popular hunting ground ever since although no other hint for GMSB or other versions of SUSY were 
found in Run I.62 78 109 101 Run II searches for GMSB are described in section 4.2.

The second major thing that came out of this observation was the clear need to be on the lookout for hints of new particles using more model-independent methods; if this event was an example of a new particle decay, then it becomes natural to speculate about what kind of particles produced it and search for other events "like it" in the hopes of providing evidence one way or the other. Unbiased follow up was difficult because, since there was no a priori search for this event, a posteriori methods had to be determined. The simplest quasi-model-independent search method used the idea that this event could have been produced by anomalous $W W \gamma \gamma$ production and decay (SM $W W \gamma \gamma \rightarrow e \nu e \nu \gamma \gamma \rightarrow e e \gamma \gamma E_{T}$ production and decay was the dominant background to this event type with $10^{-6}$ events expected). The signature-based way to look for this type of production is to consider all $\gamma \gamma$ events and search each for evidence of associated $W W$ production and decay, for example in the $W W \gamma \gamma \rightarrow(j j)(j j) \gamma \gamma$ final state. No excess in this or other $\gamma \gamma$ or $\ell+\gamma+E_{T}$ searches ${ }^{62] 78}$ t Other, more model-dependent, but still signature-based searches $\left.{ }^{82}[84] 85\right]$ also found no evidence of new physics in Run I or Run II. Ultimately, it was recognized that new, a priori methods of finding and following up on interesting events needed to found, and developed in ways that avoid potential biases. Model-independent and signature-based searches, in particular SLEUTH, which is discussed bellow, arose at the D0 experiment in Run I for these reasons (see sections 3.3 and 5.4). Ultimately, it is not clear what was the source of the event (perhaps it was a very unusual example of whatever it was), but the legacy of this event is still with us.

\subsection{Follow up on the leptoquark hints from DESY}

In 1997 the H1 $\frac{129}{12}$ and the ZEUS $\frac{130}{13}$ experiments at the DESY ep collider (HERA) reported an excess of events at high momentum transfer $Q^{2}$ with a potential explanation being the production of a single first generation scalar $L Q$ with a mass of around $200 \mathrm{GeV}$. By that time, the D0 and the CDF experiments had already

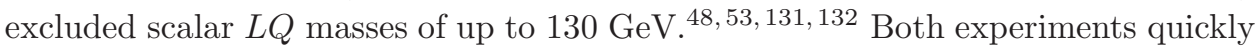
followed up on these hints in the same final state, but with $L Q$ pair production and decay and they were able to exclude first generation scalar leptoquarks in the simplest models above $200 \mathrm{GeV}$. They were then expanded to second and third generation searches, and from $L Q \rightarrow \ell q$ to include $L Q \rightarrow \nu q^{\prime}$ modes. Limits were set in both scalar and vector resonances. Ultimately, all the results were found to be consistent with the SM $58|63| 66] 76|133| 141$ These searches were extended in Run II, again with null results (see section 5.1.3).

\subsection{Signature-based searches and SLEUTH}

Signature-based searches emerged at the end of Run I. In each the analysis selection criteria are established before doing the search using systematic ways to separate any data event into a unique group based on its final state signature; specifically 
based on the set of final state particle objects. For example, those objects passing standardized lepton, photon, $E_{T}$, jet, $b$-tagging ID requirements, and above various $p_{T}$ thresholds are selected. With a clear definition of all event requirements this allows for definite predictions of the rates and kinematic properties of events from SM background processes. Note that there is no prediction of what new physics might arise, just a comparison to the SM-only hypothesis, and, consequently, there is nothing that can be optimized for sensitivity. As previously mentioned, many searches were done in Run I and Run II which followed this methodology.

The major leap forward in this area was the development of the quasi-modelindependent SLEUTH formalism at the D0 experiment ${ }^{114}$ SLEUTH traded the ability to optimize for a particular model of new physics, for breadth in covering previously unsearched territory. By looking for excesses on the tails of distributions (with a bias towards the large $Q^{2}$ interactions as it is more likely that new physics has a large scale or mass as the lower scales and masses are already well probed) SLEUTH looked for regions in the data that were not well described by the SM-only background predictions. It made a novel use of pseudoexperiments (and was a powerful early user of these methods) to quantify how unusual the largest observed deviation was. As a test, SLEUTH was able to show that it could discover $W W$ and top-quark production in the dilepton final state at many standard deviations (s.d.) in the case that neither were included in the background modeling. It was the same with leptoquarks, at a certain production level and mass, in lepton+jets. Ultimately, SLEUTH was run on $\sim 50$ final states at the D0 experiment and compared the fluctuations to expectations 118$] 142$ (see Fig. 1(b)). The distribution of the fluctuations were consistent with statistical expectations. This methodology was eventually adopted by other experiments, for example at the HERA experiments, $\frac{143}{14}$ and the CDF experiment in Run II where it was extended for the other types of systematic, model-independent search strategies (described in section 5.4). This methodology is currently less used for a number of reasons, primarily that it is not always clear how to quantify the search sensitivity to new physics.

\section{Supersymmetry}

In this section we focus on the searches for SUSY during Run II. We note that there are good reasons to expect to find sparticles at Tevatron energies, in particular since the Higgs mass would potentially diverge if there are no sparticles (most importantly the top squark, or stop for short) with a mass at or below the TeV scale. ${ }^{8}$ Until the observation of the ee $\gamma \gamma E_{T}$ candidate event in Run I, most analyses focused on mSUGRA-type models with a hierarchy of heavy colored states and a light LSP as a dark matter candidate and its smoking-gun signature of large $\mathbb{E}_{T}$. We will focus on mSUGRA in section 4.1. In section 4.2 we will discuss GMSB SUSY searches with their smoking gun final states of photons and $E_{T}$ from light gravitinos. Finally, in section 4.3 we will discuss $R$-parity violating (RPV) scenarios. Ultimately, most of these results are from the first half of Run II data as the turn on of the LHC, with its 
larger energy and production cross sections and comparable luminosities, eclipsed the Fermilab Tevatron collider sensitivity and obviated the need to get results using the full dataset. Other searches with SUSY interpretations, such as hidden-valley models, and other long-lived particles like CHAMPS, are described in section 5 Other searches, like $B_{s} \rightarrow \mu \mu$, which have important SUSY interpretations are found in the heavy flavor chapter of this review 144

\section{1. mSUGRA/Heavy LSP models}

While mSUGRA models have many theoretical advantages, from an experimental standpoint they are valued because they simplify the 128 parameter model down to four parameter d and a sign 6 which specify the sparticle masses and decay products. Equally valuable is that large chunks of the regions turn out to be qualitatively similar and much of the non-excluded parameter space has the lightest neutralino, $\tilde{\chi}_{1}^{0}$, as the LSP, which provides a cold-dark matter candidate. An important difference is between low and high $\tan \beta$; at low $\tan \beta$ the sparticles from all three generations are degenerate (or nearly degenerate) in mass, while in models with high $\tan \beta$, the third generation sparticles (stops, sbottoms and staus) can be much lighter than all other generations, leading to final states enriched with $\tau$-leptons and/or $b$-quarks.

While the couplings of the sparticles to their SM counterparts is important, perhaps the most important issue in the production of sparticles at the Fermilab Tevatron collider is their masses. If the colored objects (squarks and gluinos) are light enough, their production cross sections will dominate; if they are too heavy, they cannot be produced in significant enough quantities to be seen. Typically the gauginos are much lighter in mass so an important region of parameter space is the case where the colored objects are out of reach and gaugino pair production dominates the overall sparticle production. These two cases are again separated by high and low values of $\tan \beta$.

\subsubsection{Light flavor squarks and gluinos}

If the masses of the gluinos or the first and second generation squarks are favourable, then the large production rate of these sparticles provides a golden channel for the search for SUSY. Squarks and gluinos are expected to be produced in pairs, $\tilde{g} \tilde{g}, \tilde{g} \tilde{q}$ and $\tilde{q} \tilde{q}$, and then to decay via $\tilde{q} \rightarrow q \tilde{\chi}_{1}^{0}$ and $\tilde{g} \rightarrow q \tilde{q} \tilde{\chi}_{1}^{0}$. Each will lead to final states with jets and large $\mathbb{E}_{T}$, with the number of jets depending on the whether the squark or gluino is heavier. Alternatively, leptonic decay modes of squarks from gluino pair production (e.g. $\tilde{g} \rightarrow q \tilde{q} \rightarrow q q \tilde{\chi}_{1}^{ \pm} \rightarrow q q \ell \nu \tilde{\chi}_{1}^{0}$ ) can lead to final states with same-

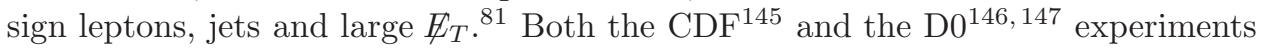

\footnotetext{
d The four mSUGRA parameters are: (i) $m_{0}$ - common mass parameter of scalars (squarks, sleptons, Higgs bosons) at the GUT scale; (ii) $m_{1 / 2}$ - common mass of gauginos and higgsinos at the GUT scale; (iii) $A_{0}$ - common trilinear coupling; and (iv) $\tan \beta$ - ratio of Higgs boson vacuum expectation values.

${ }^{\mathrm{e}} \operatorname{sign}(\mu)= \pm 1-\operatorname{sign}$ of $\mu$ SUSY conserving higgsino mass parameter.
} 
searched for evidence of these particles, with limits in different scenarios shown in Fig. 2(a,b) interpreted as limits on squarks and gluinos on the one hand and in the $m_{0}$ vs. $m_{1 / 2}$ mSUGRA parameter plane on the other. For many years these results were the most sensitive, with no substantive competition from LEP. To be complementary, a new set of high $\tan \beta$ searches emerged with squarks decaying to jets, $\tau$-leptons and large $E_{T}$ in the final state ${ }^{148}$ No evidence was observed as shown in Fig. 2(c). These were, at the time, the world's most sensitive searches.
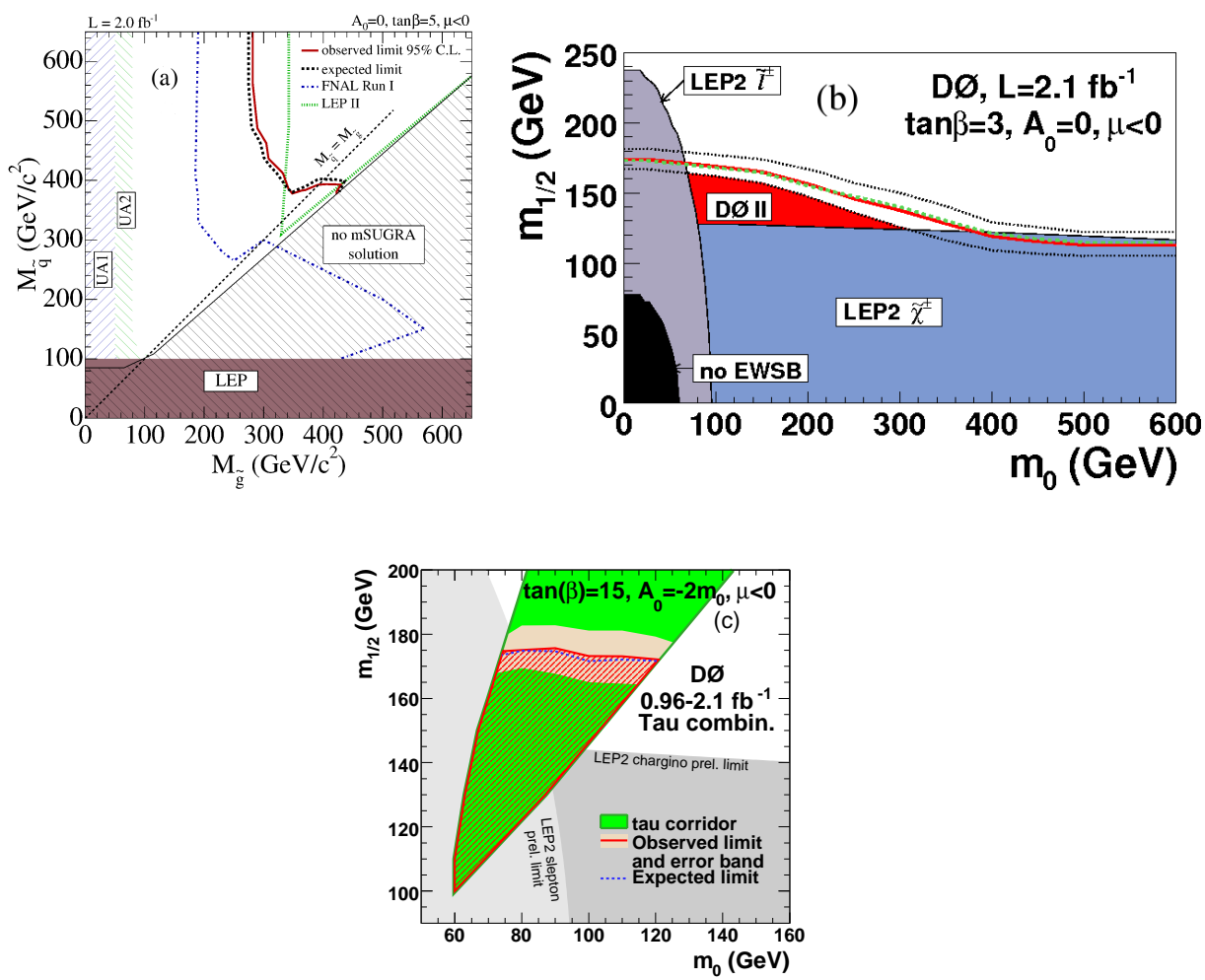

Fig. 2. (color online) Limits from the search for squarks and gluino production in the $E_{T}+$ jets final state, including jets for hadronically decaying $\tau$-lepton. Shown are the excluded region (a) in the $m_{\tilde{q}}$ vs. $m_{\tilde{g}}$ plane from the CDF experiment, and (b) in the $m_{1 / 2}$ vs. $m_{0}$ plane from the D0 experiment. (c) Limits in the $m_{1 / 2}$ vs. $m_{0}$ plane from the large $\tan \beta$ search with hadronic $\tau$-lepton combined with the jets $+E_{T}$ search from the D0 experiment.

\subsubsection{Bottom and top squarks}

The large mass difference between the third generation particles in the SM and their lighter counterparts in the first and second generations suggests that perhaps the third generation is special. This specialness can manifest itself in SUSY models with 
high $\tan \beta$ which predict that third generation squarks (and/or sleptons) are lighter than their first and second generation counterparts and decay differently, often to third generation particles like $\tau$-leptons and $b$-quarks. One strategy to search for bottom squarks is to use similar analysis techniques for light flavor squarks and gluinos searches in the jets $+E_{T}$ final state, but with the additional requirement of $b$-tagging of one or more of the jets. The simplest is direct sbottom pair production with $\tilde{b} \tilde{b} \rightarrow b \tilde{\chi}_{1}^{0} b \tilde{\chi}_{1}^{0} \rightarrow b b+E_{T}{ }^{149}[151$ Complementary searches can be done where the sbottoms are produced as the decay products of a gluino. Specifically, $\tilde{g} \tilde{g} \rightarrow$ $b \tilde{b} b \tilde{b} \rightarrow b b \tilde{\chi}_{1}^{0} b b \tilde{\chi}_{1}^{0} \rightarrow 4 b+E_{T} \cdot \frac{152] 153}{15}$ The results are shown in Fig. [3.
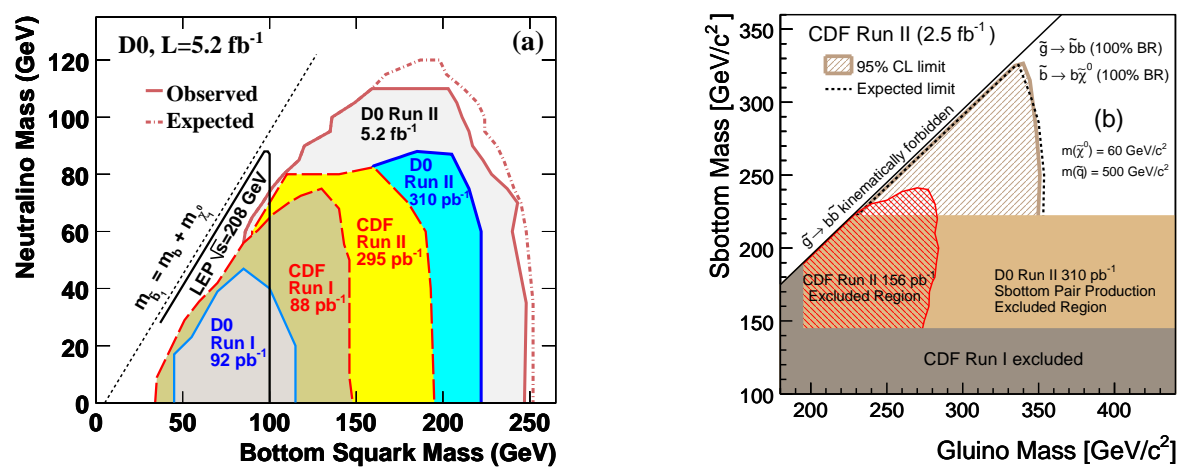

Fig. 3. (color online) (a) The excluded region in the $m_{\tilde{\chi}_{1}^{0}}$ vs. $m_{\tilde{b}}$ from the D0 experiment from the search for direct sbottom production and decay. (b) The exclusions in the $m_{\tilde{b}}$ vs. $m_{\tilde{g}}$ plane from the CDF experiment in the search for sbottoms from gluino pair production and decay.

In recent years, the case for light stops has been motivated by the need to regularize the Higgs boson mass.$^{8}$ Many more versions of stop searches are required because of the many possible decay modes of the stop. For example, the stop can decay via charged or neutral modes. In the charged modes, the stop decays via $\tilde{t} \rightarrow b \tilde{\chi}_{1}^{ \pm}$and the chargino decays via $\nu \tilde{\ell}, \ell \tilde{\nu}$ or $b W \tilde{\chi}_{1}^{0}$, where the $W$ boson is either real or virtual depending on the mass differences. In all cases, we get $\tilde{t} \rightarrow b \ell+\mathbb{E}_{T}$, but with different kinematics depending on the masses. There are multiple searches in this final state 154160 with results shown in Fig. $4(a, b)$. In the neutral modes, $\tilde{t} \rightarrow t \tilde{\chi}_{1}^{0}$ or $\tilde{t} \rightarrow c+\tilde{\chi}_{1}^{0}, 161164$ there are also a number of searches, again with null results and limits shown in Fig. प4(c).

\subsubsection{Gauginos}

While the squarks and gluinos have the largest production cross section (at given mass) it is very possible (and favored in some scenarios) that their masses are out of reach of the Fermilab Tevatron collider. Thus, a full set of searches for the lighter 

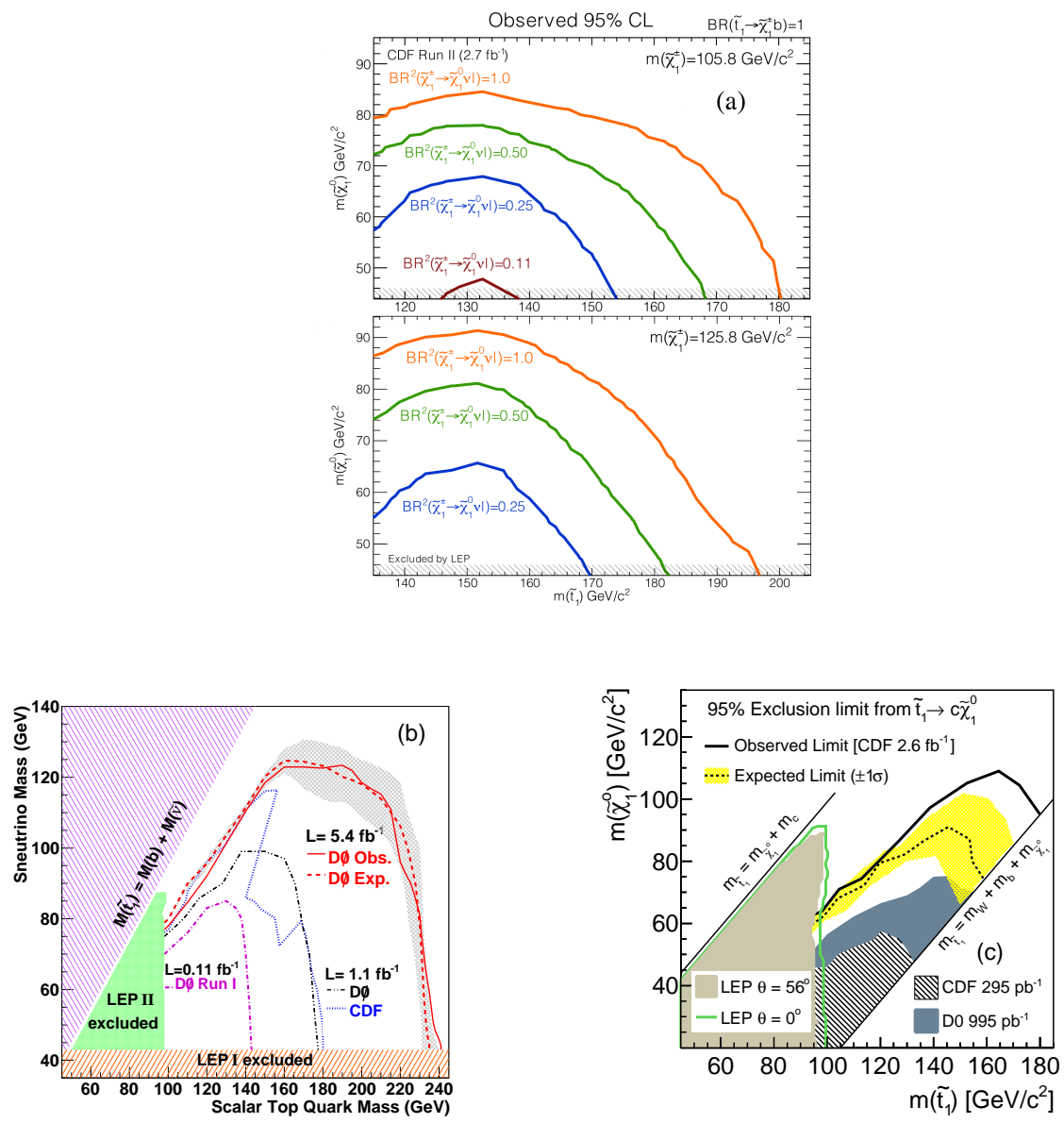

Fig. 4. (color online) Exclusion regions in the searches for stops. (a) The results in the $m_{\tilde{\chi}_{1}^{0}}$ vs. $m_{\tilde{t}}$ plane for several values of $\mathcal{B R}\left(\tilde{\chi}_{1}^{ \pm} \rightarrow \tilde{\chi}_{1}^{0} \ell \nu\right)$ and two different chargino masses form the CDF experiment. (b) The results in the $m_{\tilde{\nu}}$ vs. $m_{\tilde{t}}$ plane from the search for the $\tilde{t} \rightarrow \tilde{\chi}_{1}^{ \pm} b$ through sleptons and sneutrinos form the D0 experiment, and (c) the results in the $m_{\tilde{\chi}_{1}^{0}}$ vs. $m_{\tilde{t}}$ plane from the search for the $\tilde{t} \rightarrow \tilde{\chi}_{1}^{0} c$ from the CDF experiment.

sparticles, in particular the lightest chargino and the next-to-lightest neutralino are crucial. The golden final state modes for gaugino pair production and decay is $\ell \ell \ell+E_{T}$ (the trilepton final state $)^{165}\left[168\right.$ or two same-sign leptons $+E_{T} \underline{169 \mid 170]}$ as there are very few SM backgrounds for each. Late in Run II many of the searches included $\tau$-lepton final states to extend the searches to include higher $\tan \beta{ }^{166}$ No evidence of these sparticles have been observed at the CDF or the D0 experiments, with limits shown in Fig. 5 

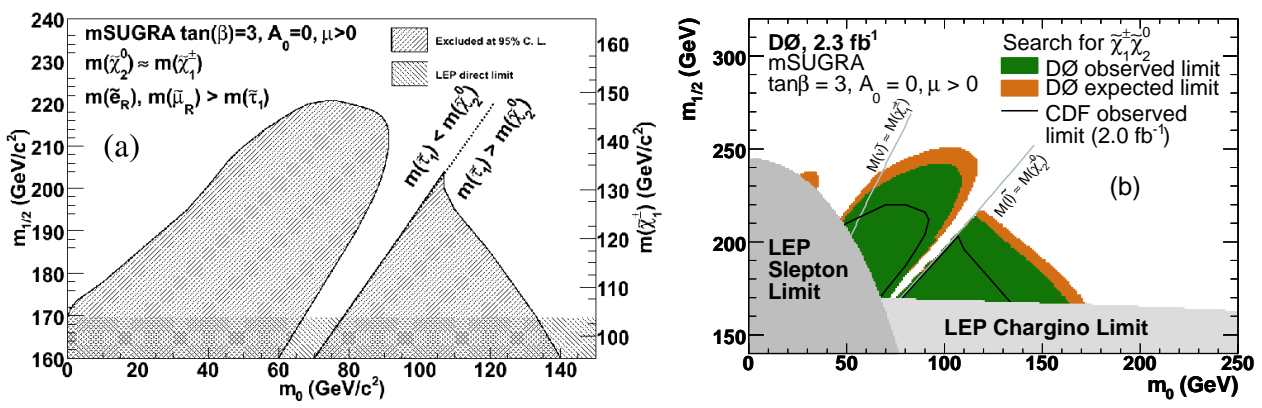

Fig. 5. (color online) Excluded region in the $m_{1 / 2}$ vs. $m_{0}$ plane in the search for charginos and neutralinos in the final state with three leptons from (a) the CDF experiment and (b) the D0 experiment.

\subsection{Gauge-mediated supersymmetry breaking (GMSB) models}

The hallmark of gauge mediated supersymmetry breaking models is the light gravitino, $\tilde{G}$, and searches for GMSB are usually (but not always) done in the context of minimal models, typically using the SPS-8 relations 171 This essentially leaves two free theory parameters: the masses (which typically assume fixed mass relations) and the lifetime $\left(\tau_{\tilde{\chi}_{1}^{0}}\right)$ of the lightest neutralino which is the next-to-lighest sparticle (NLSP). Since the parameter space where squarks and gluinos are accessible at Tevatron collider energies is easily ruled out (although not done explicitly), searches focus on the pair production of the lightest charginos and neutralinos. Through cascade decays, each chargino and/or neutralino typically decays to a $\tilde{\chi}_{1}^{0}$ or $\tilde{\tau}$ accompanied by other high $p_{T}$ SM light particles. The $\tilde{\chi}_{1}^{0}$ typically decays to $\gamma \tilde{G}$ (if the mass is low) or to $Z \tilde{G}$ if the mass is large; the $\tilde{\tau}$ decays via $\tilde{\tau} \rightarrow \tau \tilde{G}$. In all cases, the $\tilde{G}$, like the $\tilde{\chi}_{1}^{0}$ in mSUGRA models, leaves the detector and gives significant $E_{T}$. The lifetime and masses of the sparticles dictate the different final states. Specifically, in the $\tilde{\chi}_{1}^{0} \rightarrow \gamma \tilde{G}$ scenario with $\tau_{\tilde{\chi}_{1}^{0}} \leqslant 1 \mathrm{~ns}$, both $\tilde{\chi}_{1}^{0}$ decay in the detector, giving a final state of $\gamma \gamma+E_{T}+X$. For intermediate lifetimes, $1 \leqslant \tau_{\tilde{\chi}_{1}^{0}} \leqslant 50 \mathrm{~ns}$, frequently one $\tilde{\chi}_{1}^{0}$ travels a significant distance in the detector before decaying and the other leaves the detector without decaying or interacting. If this occurs, the event will be reconstructed as a $\gamma+E_{T}$ event, where the time-ofarrival of the photon at the calorimeter will be slightly later than "expected"; these photons are known as "delayed photon" $\gamma_{\text {delayed }}{ }^{[172}$ The different lifetime scenarios are considered separatelyf. For the scenario where the $\tilde{\chi}_{1}^{0}$ can decay via $\tilde{\chi}_{1}^{0} \rightarrow Z \tilde{G}$ we can have both $Z Z+E_{T}$ and $Z \gamma+E_{T}$ final states. For decays with $\tilde{\tau}$ sleptons as the intermediate sparticle, we can have multiple $\tau$-leptons and $\mathscr{E}_{T}$ in the final state.

In Run II, the CDF and the D0 experiments did a full suite of searches for

${ }^{\mathrm{f}}$ For large lifetimes, both neutralinos can leave the detector and are indistinguishable from mSUGRA scenarios. 
GMSB. The short-lifetime searches were done in the $\gamma \gamma+E_{T}$ final state $\frac{173}{177}$ and were a natural follow up to the searches done in Run I for the ee $\gamma \gamma E_{T}$ candidate event. In the scenarios with the intermediate $\tau_{\tilde{\chi}_{1}^{0}}$, the CDF experiment used the electromagnetic calorimeter timing readout system installed in Run II, 178 and searches were done in the $\gamma_{\text {delayed }}+$ jet $+E_{T}$ final state 179$] 180$ The D0 experiment did the first search in $Z \gamma+E_{T} 181$ and the CDF experiment did a search with same-sign $\tau$ leptons $+E_{T}, \frac{170}{170}$ No evidence was observed and limits are shown in Fig. 6. Recently, scenarios with a light neutralino and gravitino (with all other sparticles out of the reach of colliders) have been proposed, 182 and searches for this final state (without limits) have been done at the CDF experiment, in the exclusive $\gamma_{\text {delayed }}+\mathbb{E}_{T}$ with no evidence for new physics 183
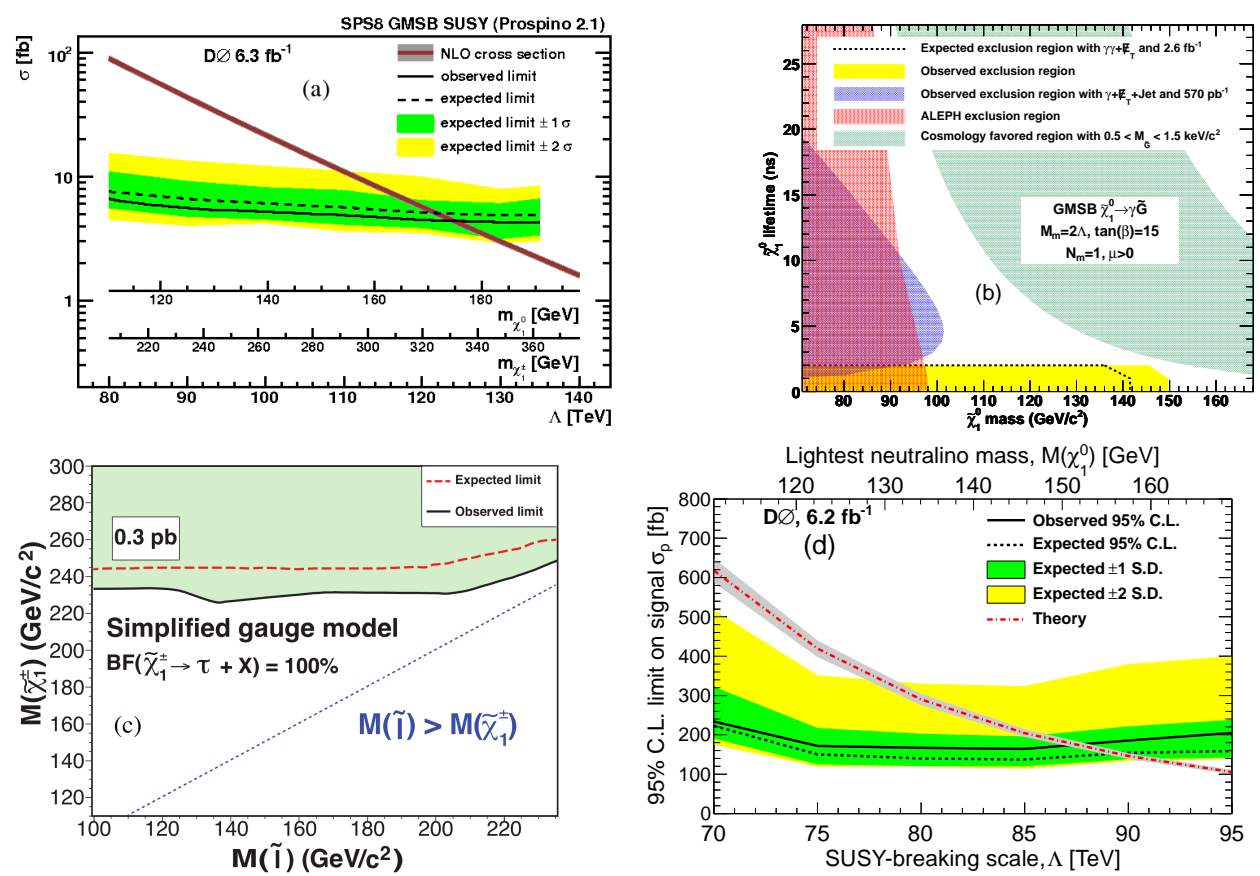

Fig. 6. (color online) Limits on GMSB scenarios. (a) The 95\% C.L. cross section upper limits from the $\gamma \gamma+E_{T}$ final state as a function of scale $\Lambda, m_{\tilde{\chi}_{1}^{0}}$ and $m_{\tilde{\chi}_{1}^{ \pm}}$from the D0 experiment. (b) The excluded regions in the $\tau_{\tilde{\chi}_{1}^{0}}$ vs. $m_{\tilde{\chi}_{1}^{0}}$ plane from the CDF experiment from the $\gamma \gamma+E_{T}$ and $\gamma_{\text {delayed }}+E_{T}+$ jet searches. (c) The excluded region in the $m_{\tilde{\chi}_{1}^{ \pm}}$vs. $m_{\tilde{l}}$ plane from the CDF experiment in the search with $\tau$-leptons $+E_{T}$, and (d) the $95 \%$ C.L. cross section upper from $Z \gamma+E_{T}$ production as a function of $\Lambda$ and $m_{\tilde{\chi}_{1}^{0}}$ from the D0 experiment. 


\subsection{R-parity violation}

While one of the most attractive features of SUSY is its potential to solve the dark matter problem, there is no inherent requirement for $R$-parity to be conserved. If the restriction that $R$-parity conservation is released, then there is a great deal of variety in the new final states allowed from sparticle production. For pragmatic reasons, efforts focused on two different modes. The first was on pair production and decay of stops via $\tilde{t} \rightarrow b \tau^{\underline{184}}$ by the CDF experiment with limits shown in Fig. T(a). However, with the restriction of $R$-parity removed, sparticles are no longer required to be produced in pairs. An example signal of this type is single sneutrino production which decays via the $\tilde{\nu} \rightarrow e \mu, e \tau$ or $\mu \tau$ final states. A variety of searches were done at the $\mathrm{CDF} \mathrm{F}^{185]}[186$ and the $\mathrm{D} 0 \underline{187][188}$ experiments. No new physics was observed and limits were set with the results shown in Fig. 7(b,c). Other searches for RPV 189 also did not show any excess. We also note that these same results can be interpreted in terms of other models, for example lepton flavor violating $Z^{\prime}$ boson production and decay, and are described in section 5.1.1.

\section{Other BSM Searches}

We next present results on both the classic Tevatron searches as well as a number of new types of searches that originated after the beginning of Run II. These include resonances, hidden-valley model particles, long-lived particles, extra dimensions, dark matter, as well as signature-based and model-independent searches. Many of these models, like those containing an extended Higgs sector, are discussed in more detail in the Higgs boson chapter of this review, 191 although some are referenced here for completeness.

\subsection{Resonances}

One of the primary analysis techniques to search for new particles, which was developed long before the advent of colliders, is to look for resonances in the invariant mass distribution of two final state particles. This method can be used for a large number of different final states and a signature of this type can arise from new fermions and gauge bosons, excited fermions, leptoquarks, technicolor particles, and other models. These results are presented next.

\subsubsection{New fermions and bosons}

While there are many different models that predict new fermions from extending the number of generations in the SM the experiments focused on searches for different types of heavy quarks that decay to a vector boson, $V=W, Z$, and a SM quark. The CDF experiment searched for pair production and decay of fourth generation $b^{\prime}$ quarks that decay exclusively via $b^{\prime} \rightarrow b Z !^{192}$ The analysis was done in the $\ell \ell+3$ jets final state. No significant excess is observed and $b^{\prime}$ quarks are excluded with 

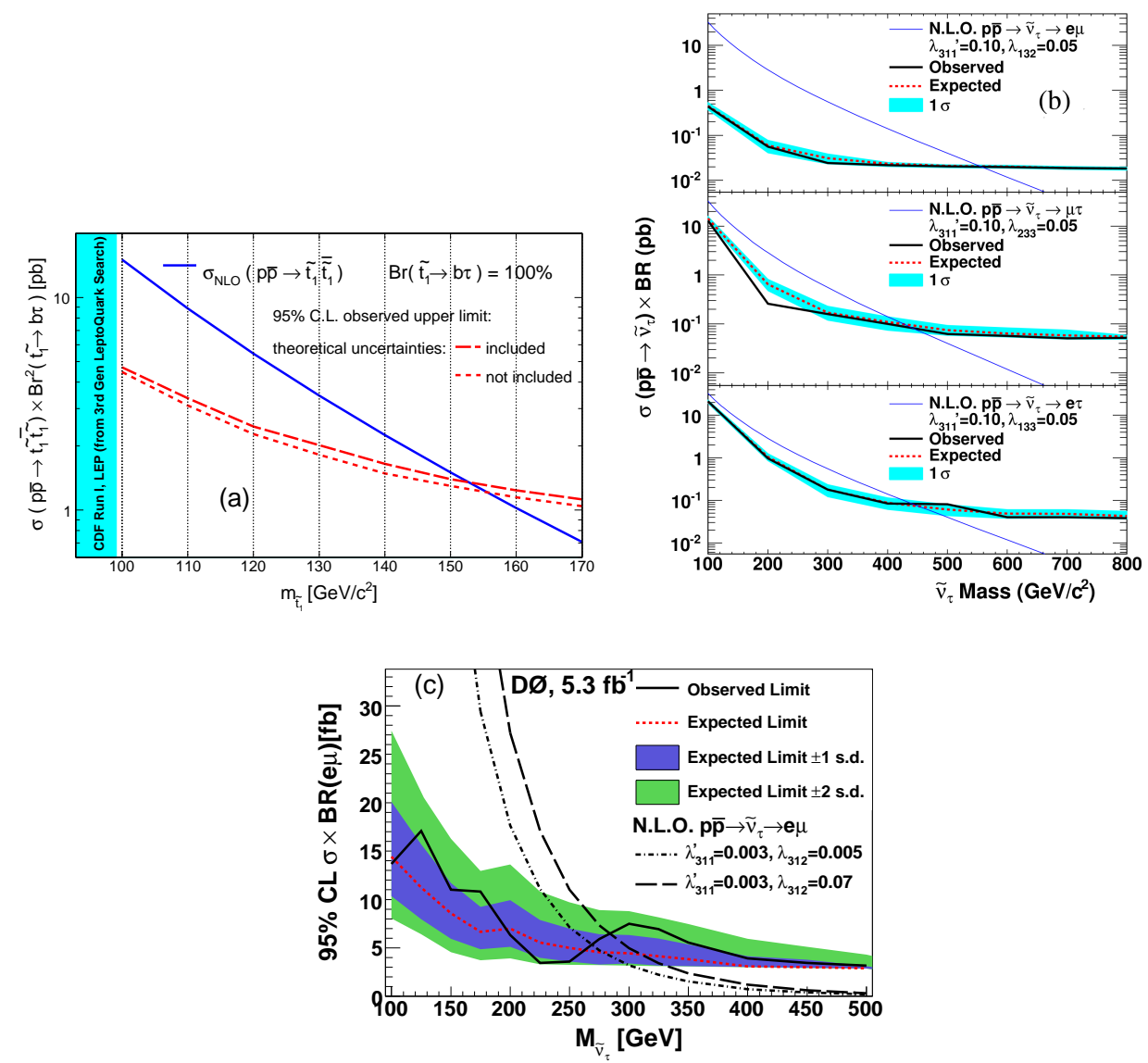

Fig. 7. (color online) Results from $R$-parity violation SUSY searches. (a) The 95\% C.L. cross section upper limit on pair-production and decay of $\tilde{t} \rightarrow b \tau$ from the CDF experiment. Cross section limit results on (b) $\tilde{\nu}$ in the $\tilde{\nu} \rightarrow e \mu, e \tau, \mu \tau$ final states from the CDF experiment and (c) $\tilde{\nu} \rightarrow e \mu$ from the D0 experiment.

$m_{b^{\prime}}<268 \mathrm{GeV}$ at $95 \%$ C.L. (see Fig. 8(a)). Another analysis by the D0 experiment searches for vector-like quarks, $Q$, in single quark electroweak production in association with SM quarks. ${ }^{193}$ At hadron colliders, electroweak production of vector-like quarks can be significant, but depends on $m_{Q}$ and the coupling strength between the $Q$ and SM quarks, $\tilde{\kappa}_{q Q}$. Single production and decay of $p \bar{p} \rightarrow q Q \rightarrow q(V q)$ can produce an excess of events in the $V+2$ jet final state. Limits are set as a function of the various model parameters; for $\tilde{\kappa}_{q Q}=1$ the process $Q q \rightarrow W q q$ is excluded for a mass $m_{Q}<693 \mathrm{GeV}$ (see Fig. 8(b)), and the process $Q q \rightarrow Z q q$ is excluded for a mass $m_{Q}<449 \mathrm{GeV}$ at $95 \%$ C.L. Other searches for fourth generation quark pair production include decays to top quarks 194200 These encompass $b^{\prime} \rightarrow t W$, 
$t^{\prime} \rightarrow W b$ and $W q$. Similar searches for a new heavy particle $T \rightarrow t+X$ where $X$ is an invisible particle found no evidence of new physics (see Fig. $8(\mathrm{c}, \mathrm{d})$ ).
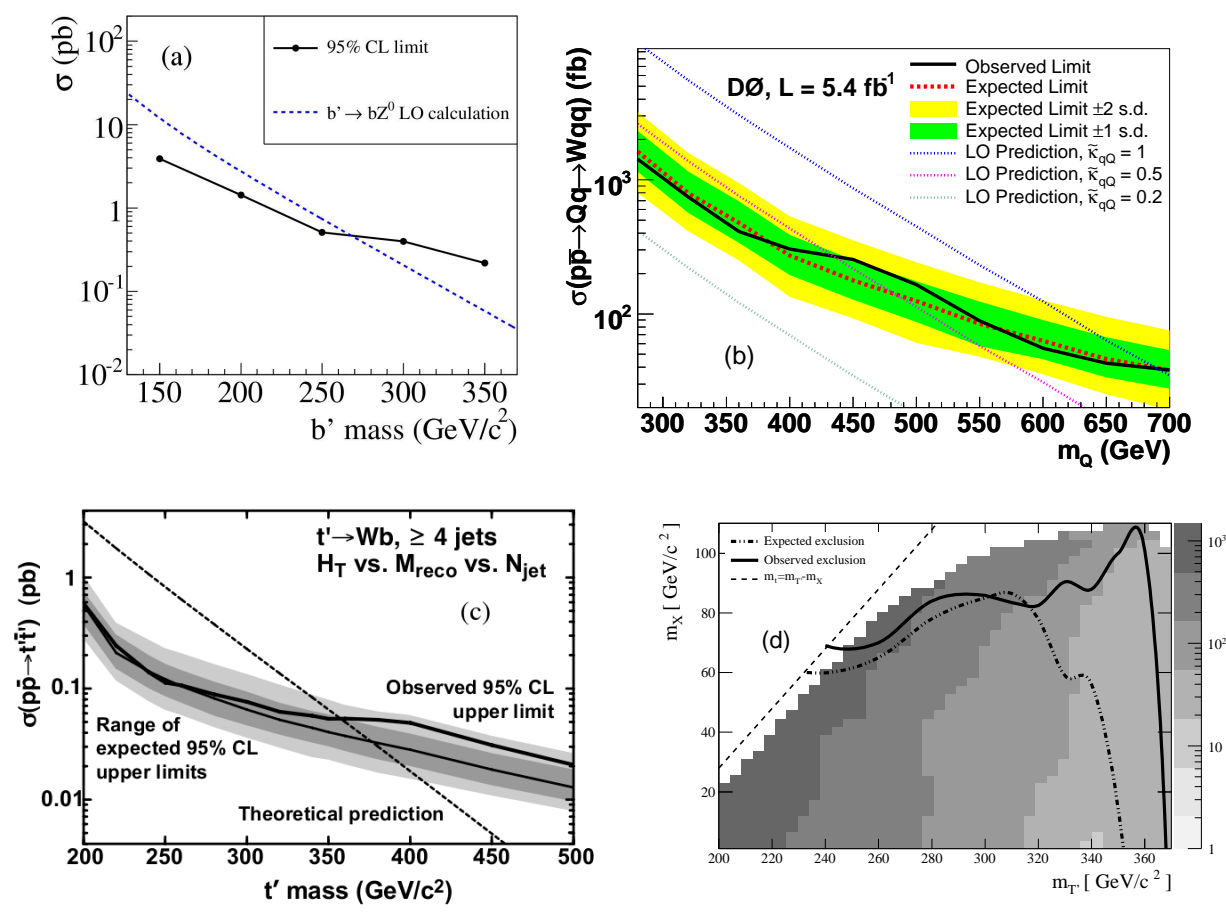

Fig. 8. (color online) (a) The 95\% C.L. cross section upper limits on pair production and decay of the $b^{\prime} \rightarrow Z b$ as a function of $m_{b^{\prime}}$ from the CDF experiment, (b) the limits on a vector-like quark, $Q \rightarrow W+$ jet as a function of $m_{Q}$ and for different coupling strengths with SM quarks, $\tilde{\kappa}_{q Q}$, from the D0 experiment, (c) the limits on the $t^{\prime} \rightarrow W b$ as a function of $m_{t^{\prime}}$, and (d) the limits in $m_{T}$ vs. $m_{X}$ in the search for a new heavy particle $T$ from the CDF experiment.

The new gauge bosons predicted in left-right symmetric models $\left(S U(2)_{L} \times\right.$ $S U(2)_{R}$ ), grand unified theories (e.g. $E_{6}$ ), or by the introduction of gauge groups beyond the SM are typically referred to as the $W^{\prime}$ or $Z^{\prime}$ bosons. Both the CDF and the D0 experiments searched for $W^{\prime}$ bosons in many different final states including $W^{\prime} \rightarrow \ell \nu, t b$ and $W Z$. The most common searches are the $W^{\prime} \rightarrow e \nu^{201 / 202}$ and $W^{\prime} \rightarrow \mu \nu \sqrt{203}$ channels and no excess of events is observed. With the assumption that the $W^{\prime} \rightarrow W Z$ mode is suppressed and that any additional generation of fermions can be ignored, the $W^{\prime}$ boson is excluded for a mass $m_{W^{\prime}}<1.12 \mathrm{TeV}$; the results are shown in Fig. 9(a,b). Additional searches for $W^{\prime} \rightarrow t t^{204} 207$ show no hints of new physics (see Fig. 9(c,d)). Searches in the diboson final state are described with other diboson results below.

A new $Z^{\prime}$ boson will occur in theories where BSM gauge groups have an ad- 

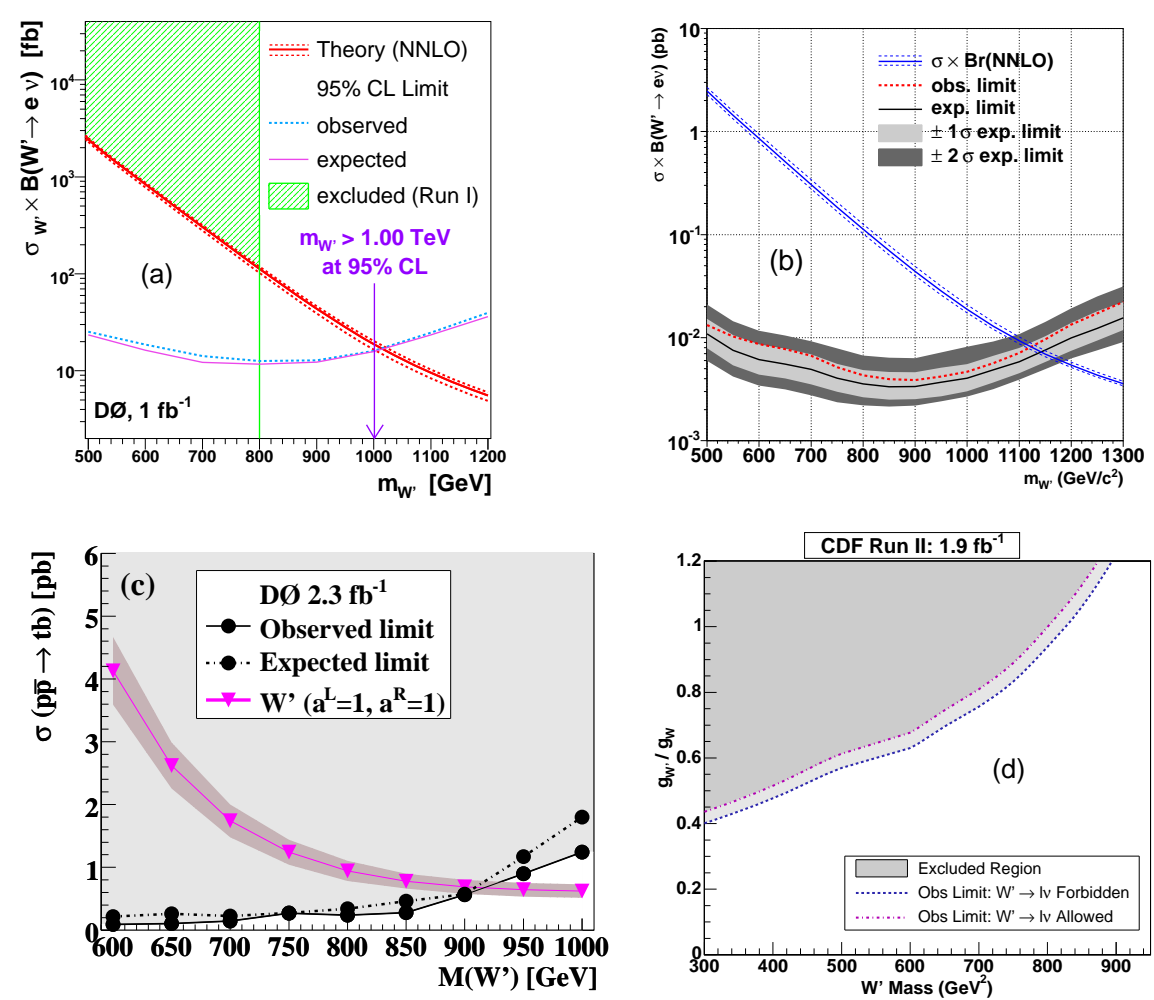

Fig. 9. (color online) The 95\% C.L. cross section upper limit on $W^{\prime} \rightarrow e \nu$ process as a function of the $M_{W^{\prime}}$ from (a) the D0 and (b) the CDF experiments. The $95 \%$ C.L. cross section upper limit on $W^{\prime} \rightarrow t b$ process (c) as a function of the $M_{W^{\prime}}$ from the D0 experiment, and (d) in $g_{W^{\prime}} / g_{W}$ vs. $M_{W^{\prime}}$ from the CDF experiment.

ditional $U(1)$ gauge group. The most common analysis is to search for a narrow resonance in the mass distribution for the $Z^{\prime} \rightarrow \ell \ell, j j, t \bar{t}$ or $W W$. Both the $\mathrm{D} 0^{208}$ and the $\mathrm{CDF} 209 \sqrt{211}$ experiment looked for these signatures in dilepton final states. Fig. 10(a) shows the $M_{e e}$ distribution from the CDF experiment, exhibiting a modest excess of events in data around $M_{Z^{\prime}} \sim 240 \mathrm{GeV}$; if only SM physics is assumed in the search region, this excess has a significance of 2.5 s.d. The D0 experiment did not observe any significant excess as shown in Fig. 10(b), and 95\% C.L. upper limits on $\sigma \times B R\left(p \bar{p} \rightarrow Z^{\prime} \rightarrow e e\right)$ for various models are set, varying between $M_{Z^{\prime}}<772 \mathrm{GeV}$ and $M_{Z^{\prime}}<1023 \mathrm{GeV}$ as shown in Fig. 10(c). In both $Z^{\prime} \rightarrow \mu \mu$ searches, no significant excess was observed, with limits on production of the $Z^{\prime}$ boson assuming various models between $M_{Z^{\prime}}<817 \mathrm{GeV}$ and $M_{Z^{\prime}}<1071 \mathrm{GeV}$, as shown in Fig. 10(d). Other searches in $Z^{\prime} \rightarrow j j$ and $t \bar{t}$ found no excesses 212 215 (see Fig. 10(e,f)). The decay $Z^{\prime} \rightarrow W W$ is described with the other diboson searches below. Lepton flavor violating searches, for example $Z^{\prime} \rightarrow e \mu, e \tau, \mu \tau$ are typically 
done in the context of $R$-parity violating SUSY, but have $Z^{\prime}$ interpretations $\frac{185}{1 .}$
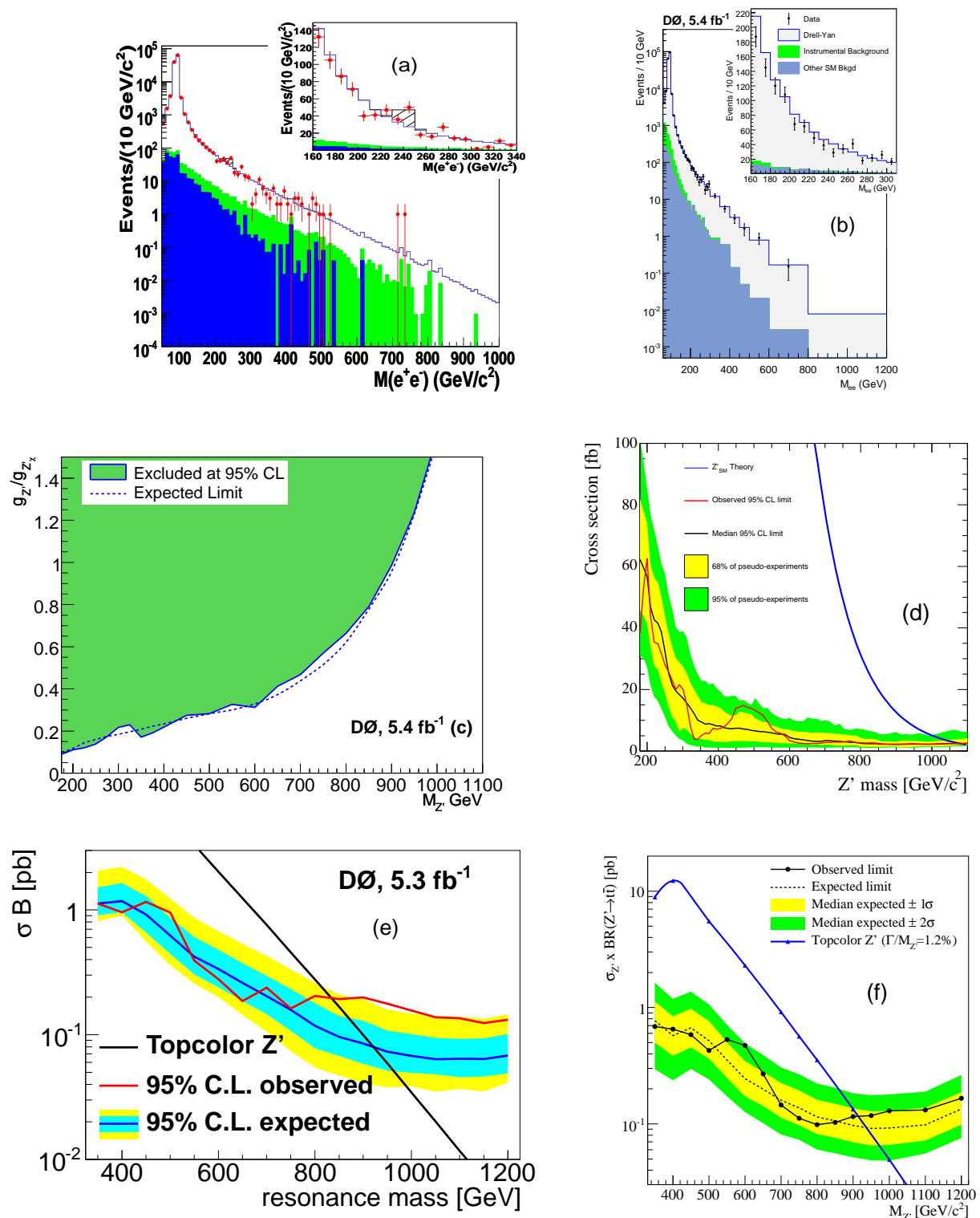

Fig. 10. (color online) The dielectron invariant mass in the search for $Z^{\prime} \rightarrow e e$ from (a) the CDF experiment and (b) the D0 experiments. (c) The 95\% C.L. upper limits on the Z' couplings ratio $\left(g_{Z^{\prime}} / g_{Z_{\chi}^{\prime}}\right)$ as a function of $M_{Z^{\prime}}$ from the D0 experiment. (d) The $95 \%$ C.L. upper limits on the $\sigma \times \mathcal{B R}\left(Z^{\prime} \rightarrow \mu \mu\right)$ as a function of the $M_{Z^{\prime}}$ from the CDF experiment. The $95 \%$ C.L. upper limits on the $\sigma \times \mathcal{B R}\left(Z^{\prime} \rightarrow t t\right)$ as a function of the $M_{Z^{\prime}}$ from (e) the D0 experiment and (f) the CDF experiments. 
The CDF and the D0 experiments also searched for resonances in the $W W$, $W Z$ and $Z Z$ decay modes. While these searches can be analyzed as $Z^{\prime} \rightarrow W W$, $Z^{\prime} \rightarrow Z Z$ and $W^{\prime} \rightarrow W Z$, other interpretations are possible. These are done at the CDF experiment in $V^{\prime} \rightarrow V V \rightarrow \ell+E_{T}+$ jets, $\stackrel{216}{2}$ and $X \rightarrow Z Z^{217}$ with various $Z$ boson decays. The $X \rightarrow Z Z$ search showed a small excess in data in the low-sensitivity four-lepton channel, which was not observed in the other two more sensitive $\ell \ell j j$ and $\ell \ell+E_{T}$ final states,, 217 this result will be interpreted in the section 5.3. The D0 experiment 218 219] searched in a combined way for $W^{\prime}$ decay to one, two or three leptons (assuming $W^{\prime} \rightarrow W Z \rightarrow \ell \ell \ell E_{T}, \ell \ell j j, \ell \ell E_{T}+$ jets). In addition to the standard methods, a novel method is used (now adopted by the LHC) to investigate the possibility that the heavy $W^{\prime}$ or $Z^{\prime}$ boson is so massive that the decay bosons are highly boosted. For hadronically decaying vector bosons the two light quarks could get merged and produce a single broad jet with an invariant mass close to the $W$ or $Z$ boson mass. No significant excess over data was found in either experiment in any of these modes. Limits on the $W^{\prime}$ boson are between $180 \mathrm{GeV}$ and $690 \mathrm{GeV}$, and on the $Z^{\prime}$ boson between $242 \mathrm{GeV}$ and $544 \mathrm{GeV}$ as shown in Fig. 11
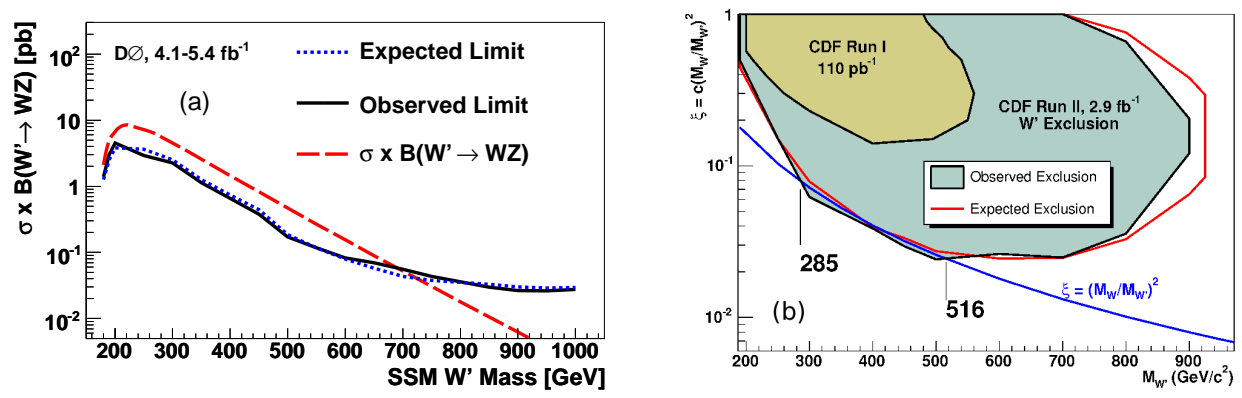

Fig. 11. (color online) (a) The 95\% C.L. upper limit on $\sigma \times \mathcal{B R}\left(W^{\prime} \rightarrow W Z\right.$ ) from the D0 experiment and (b) the exclusion region in the $\xi$ vs. $M_{W^{\prime}}$ plane in the $W^{\prime} \rightarrow W Z$ search from the $\mathrm{CDF}$ experiment, where the parameter $\xi$ sets the coupling strength between the SM $W$ boson and any new $W^{\prime}$ boson.

Finally, the CDF experiment ${ }^{220}$ searched for both resonant and non-resonant production of pairs of strongly interacting particles, each of which decays to a pair of jets, $p \bar{p} \rightarrow X \rightarrow(Y Y) \rightarrow(j j)(j j)$ and $p \bar{p} \rightarrow Y Y \rightarrow(j j)(j j)$. This search is particularly sensitive at lower masses, where the LHC experiments expect high background rates. No evidence of new particles is observed and results are interpreted as an exclusion of the $Y$ particle in both production scenarios with the results shown in Fig. 12 These results are directly applicable in axigluon models. 

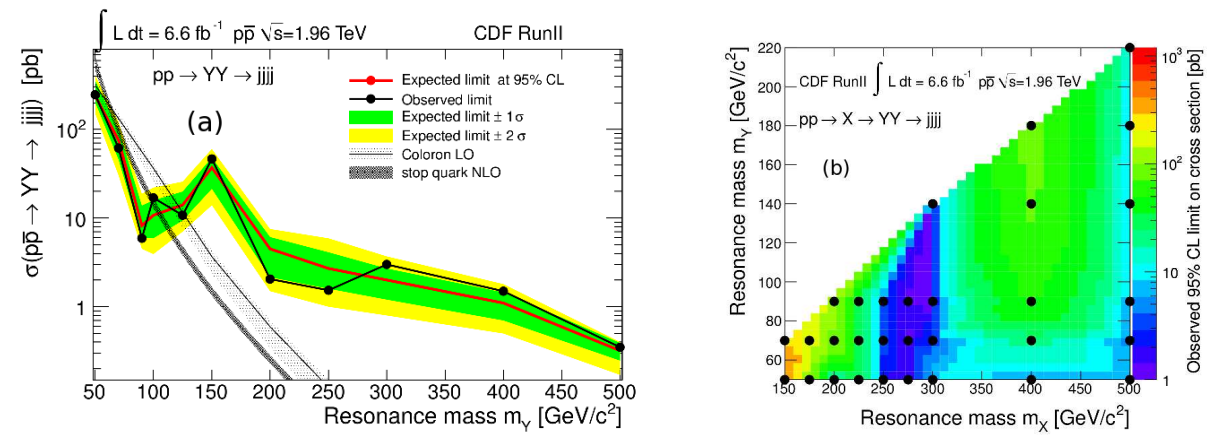

Fig. 12. (color online) The 95\% C.L. upper limits on (a) $\sigma(p \bar{p} \rightarrow Y Y \rightarrow j j j j$ ) as a function of the $M_{Y}$ and (b) $\sigma(p \bar{p} \rightarrow X \rightarrow Y Y \rightarrow j j j j)$ in the $M_{Y}$ vs. $M_{X}$ plane from the CDF experiment.

\subsubsection{Excited fermions}

The search for compositeness focuses on excited states of the SM fermions. Both excited electrons and excited muons, as well as excited quarks are searched for in

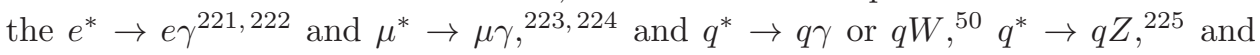
$q^{*} \rightarrow q g$ modes ${ }^{212}$ Results are interpreted as exclusion limits in contact interaction model for a mass $m<876(853) \mathrm{GeV}$ for $e^{*}\left(\mu^{*}\right)$, and in a gauge-mediated model for a mass $m<430(410) \mathrm{GeV}$ for $e^{*}\left(\mu^{*}\right)$.

\subsubsection{Leptoquarks}

Leptoquarks can exist as either vectors or scalars and can be produced in either pair production or single production modes. In all cases, the $L Q$ can decay to $\ell q$ or $\nu q^{\prime}$ where $\ell=e, \mu, \tau$, and the parameter $\beta$ defines the branching fraction for $L Q \rightarrow \ell q$. Due to experimental constraints on flavour changing neutral currents,, 9 it is assumed that $L Q \mathrm{~s}$ only couple to fermions of the same generation. Both the $\mathrm{CDF}^{226} \sqrt{229}$ and the $\mathrm{D} 0$ 151]230 239 experiments focused on pair production of leptoquarks of all generations in the $\ell q \ell q, \ell q \nu q^{\prime}$ and $\nu q \nu q$ final states. In all cases, no excesses were observed. Since vector and scalar production are similar, limits can be set on both using the same results, but we begin by reporting the results in the scalar $L Q$ case. For $\beta=0$, when both $L Q$ decay to $\nu q$, it is not possible to distinguish between the search for the first and second generation, and a common limit for a mass $m_{L Q}<214 \mathrm{GeV}$ is set; $b$-tagging allows for a specific search for the third generation in $b b+E_{T}$, and the limit is $m_{L Q}<247 \mathrm{GeV}$. Searches with charged leptons are done in the $\ell+$ jets $+E_{T}$ and $\ell \ell+$ jets final states; the third generation search often requires the hadronic decays of $\tau$-lepton accompanied with $b$-jets. For $\beta=0.5$ the first generation $L Q$ is excluded for a mass $m_{L Q}<326 \mathrm{GeV}$, the second generation for a mass $m_{L Q}<270 \mathrm{GeV}$, and the third generation for a mass $m_{L Q}<207 \mathrm{GeV}$; for $\beta=1$ the first generation $L Q$ is excluded for a mass $m_{L Q}<199 \mathrm{GeV}$, the second generation for a mass $m_{L Q}<316 \mathrm{GeV}$, and the third generation for a mass 
$m_{L Q}<210 \mathrm{GeV}$. Figure13(a) shows the exclusion region in the $\beta$ vs. $M_{L Q}$ plane in the search for the first generation scalar $L Q$ pairs. Reinterpreting the data in terms of first generation vector $L Q$ model, Fig. 13(b) shows the limits for three different assumptions about the couplings. For the third generation, with $\beta=1$, vector $L Q \mathrm{~s}$ are excluded for a mass $m_{L Q}<317 \mathrm{GeV}$ and $m_{L Q}<251 \mathrm{GeV}$ at $95 \%$ C.L. with two different assumptions about couplings 226
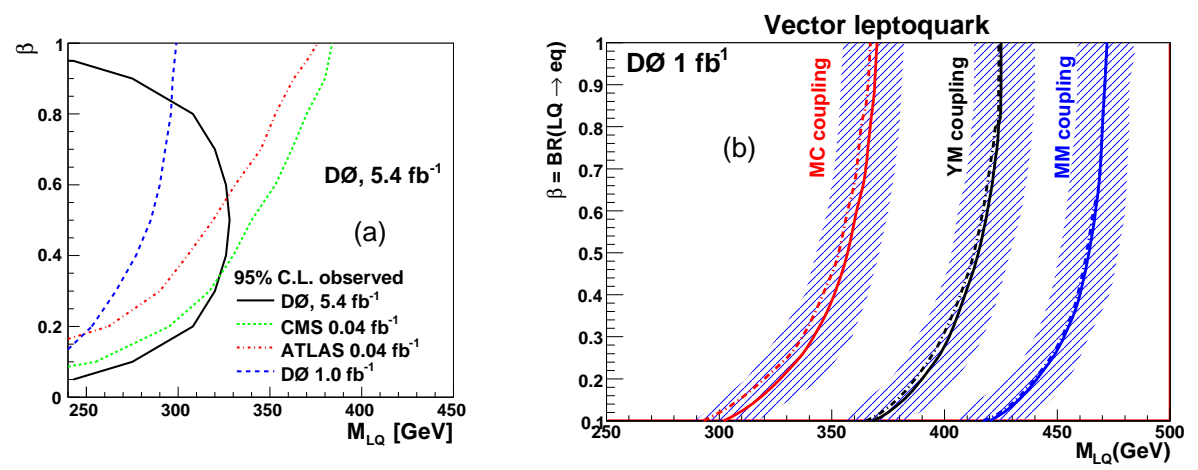

Fig. 13. (color online) (a) The exclusion region in the $\beta$ vs. $M_{L Q}$ plane from the search for first generation scalar $L Q$ pairs. (b) The exclusion regions in the same plane but for first generation vector $L Q$ pairs. Both results are from the D0 experiment.

\subsubsection{Technicolor}

Much of technicolor phenomenology is driven by the technicolor strawman model ${ }^{24}$ In this model, the most promising signature is the production and decay of a technicolor $\rho_{T}$, which can decay via $\rho_{T} \rightarrow \pi_{T}+W \rightarrow b b W$ (where $\pi_{T}$ is a technipion) or $\rho_{T} \rightarrow W Z$ depending on the masses of the particles involved. Other new particles such as a techniomega, $\omega_{T}$, can be produced and decay via $\omega_{T} \rightarrow \gamma+\pi_{T} \rightarrow \gamma b b$. No evidence for new physics is observed!218 240|241 Some of the expected and observed 95\% C.L. excluded regions are shown in Fig. 14.

\subsubsection{Other resonance searches}

We next mention a few resonance searches that do not fall in to any of the above categories. The SM predicts the branching ratio $Z \rightarrow \pi^{0} \gamma$ to be between $10^{-12}$ and $10^{-9}$. The CDF experiment ${ }^{242}$ searched for this rare process by looking for a narrow resonance with $m \sim 90 \mathrm{GeV}$ in the $\gamma \gamma$ invariant mass spectrum; This search is extended to include the quantum mechanically forbidden processes $Z \rightarrow \gamma \gamma$ and $Z \rightarrow \pi^{0} \pi^{0}$. No significant excess in the data was found, and $95 \%$ C.L. upper bounds on the branching ratios are determined: $\mathcal{B R}\left(Z \rightarrow \pi^{0} \gamma\right)<2.01 \times 10^{-5}$, 

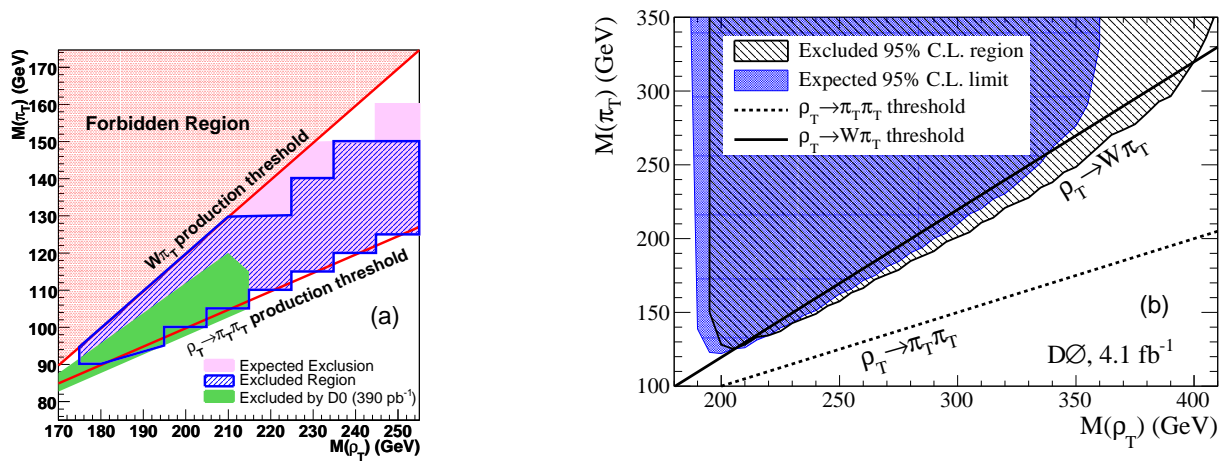

Fig. 14. (color online) Results for technicolor models. (a) The exclusion region in the $M_{\pi_{T}}$ vs. $M_{\rho_{T}}$ plane from the $b b+W$ final state from the CDF experiment and (b) the results for the $\rho_{T} \rightarrow W Z$ final state from the D0 experiment.

$\mathcal{B R}(Z \rightarrow \gamma \gamma)<1.46 \times 10^{-5}$, and $\mathcal{B R}\left(Z \rightarrow \pi^{0} \pi^{0}\right)<1.52 \times 10^{-5}$, which remain most stringent in the world.

In 2011 the CDF experiment ${ }^{29}$ created a world-wide stir when it reported an excess of events in the invariant mass distribution of jet pairs produced in association with a $W$ boson in the leptonic final state. The observed excess, with a dijet invariant mass between 120 and $160 \mathrm{GeV}$, was at the 3.2 s.d. level, appeared to have a Gaussian shape (as expected from a new particle due to mass resolution effects) and gave a production cross section at the $4 \mathrm{pb}$ level as shown in Fig.15(a,b). Following this lead, the D0 experiment ${ }^{243}$ investigated this final state, but no excess was found as shown in Fig. 15(c); limits were set that excluded new particle production above $1.9 \mathrm{pb}$. The final resolution of this potential excess came when the CDF experiment published the result in the jets $+E_{T}$ final state ${ }^{244}$ and an updated version ${ }^{245}$ of the leptonic analysis, where a number of systematic effects were investigated and taken into account including improved understanding of the detector response to quarks and gluons separately, and modeling of instrumental backgrounds. In these searches there is no indication of an excess and the final results are shown in Fig. 15(d). The $95 \%$ upper limit was set on the production cross section of the new particle at $0.9 \mathrm{pb}$. This story underscores the need for two experiments.

\subsection{Hidden-valley models, CHAMPS and other long-lived particles}

There are many different types of long-lived particles predicted in new models. A few have already been described in the GMSB section, but there are others such as hidden-valley model particles, CHAMPS (typically in SUSY models), monopoles, stopped gluinos and quirks which are described next. 

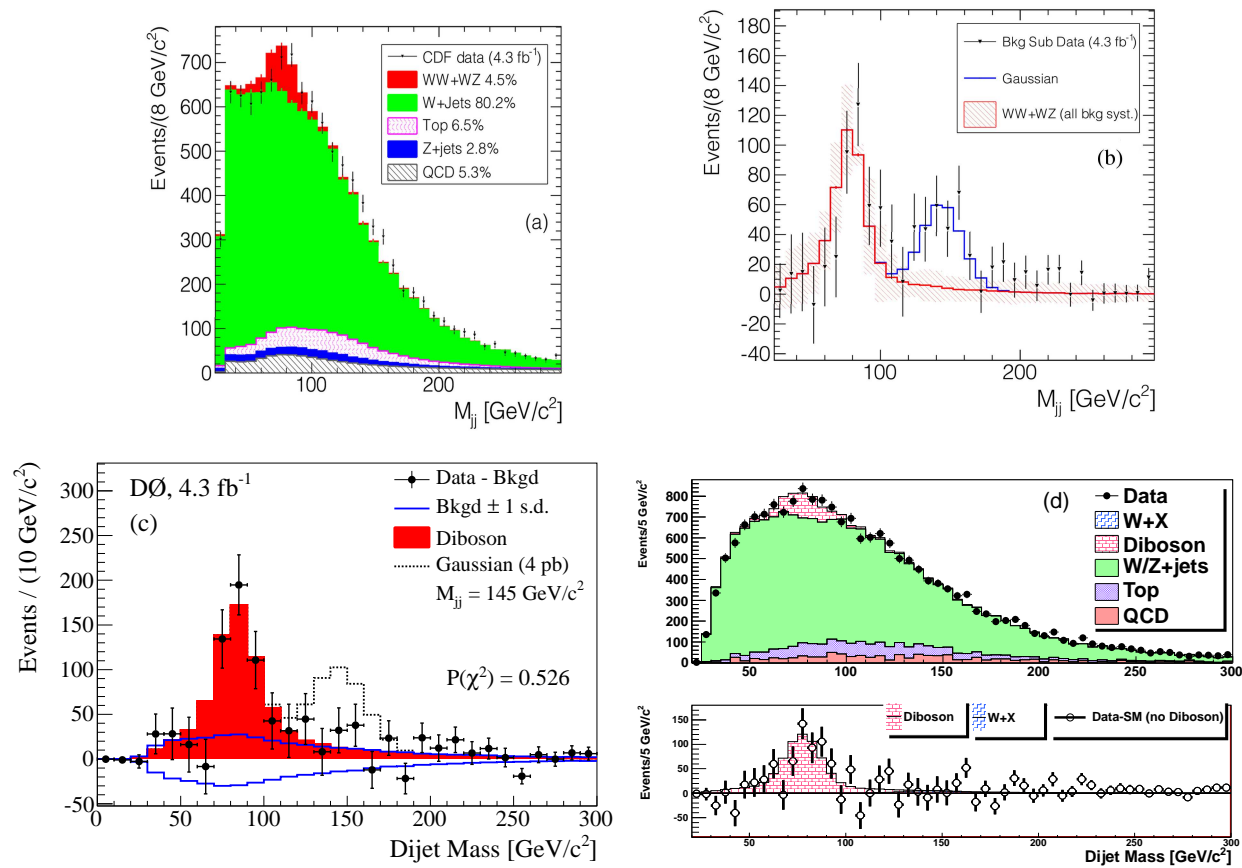

Fig. 15. (color online) The dijet invariant mass in $W+$ dijet events in the $\ell+E_{T}+2$ jet final state: (a) the data plotted on top of the known SM processes showing an excess around $140 \mathrm{GeV}$, and (b) the same data with the SM backgrounds (except $W W$ and $W Z$ ) subtracted, and using a Gaussian component fit for the excess region from the CDF experiment. (c) The results in the same final state from the D0 experiment. (d) The updated CDF analysis showing that the original excess was due to detector and analysis effects.

\subsubsection{Hidden-valley/dark photons}

Hidden valley $(\mathrm{HV})$ models provide a framework for studying the phenomenology of secluded sectors, but make no specific predictions. The D0 experiment performed variety of different searches. The first analysis 246 searched for Higgs boson production and decay into a pair of neutral long-lived HV particles that each decay to a $b \bar{b}$ pair. The search is for pairs of very-displaced vertices in the tracking detector, with radii in the range between $1.6-20 \mathrm{~cm}$ from the beam axis. No excess is found and limits are set as shown in Fig 16(a).

HV models can also include SUSY. In one search gaugino pairs can be produced and decay into HV particles, in particular a new light gauge boson (known as a dark photon) which in turn decays via fermion pairs, and HV (or dark) neutralinos which escape the detector and produce large $E_{T}$. This final state includes a photon, two spatially close leptons and large $E_{T}$. Since there is no evidence of dark photons, limits are set ${ }^{247}$ and the results are shown in Fig. 16(b). A complementary search for gaugino pair production is done by searching for a pair of dark photons, a pair of dark neutralinos and other SM particles in the final state. These events have the 
unique final state of a pair of isolated "jets" of charged leptons, so-called leptonic jets, produced in association with a large amount of $\mathbb{E}_{T}$. Again, no evidence was found $\frac{248}{2}$ and limits are shown in Fig. 16)(c). Finally, searches can be done for longlived particles in $\mathrm{HV}$ with pairs of electrons or photons in the final state with results from $b^{\prime} \rightarrow Z q \rightarrow e e q^{249}$ shown in Fig. 16)(d).
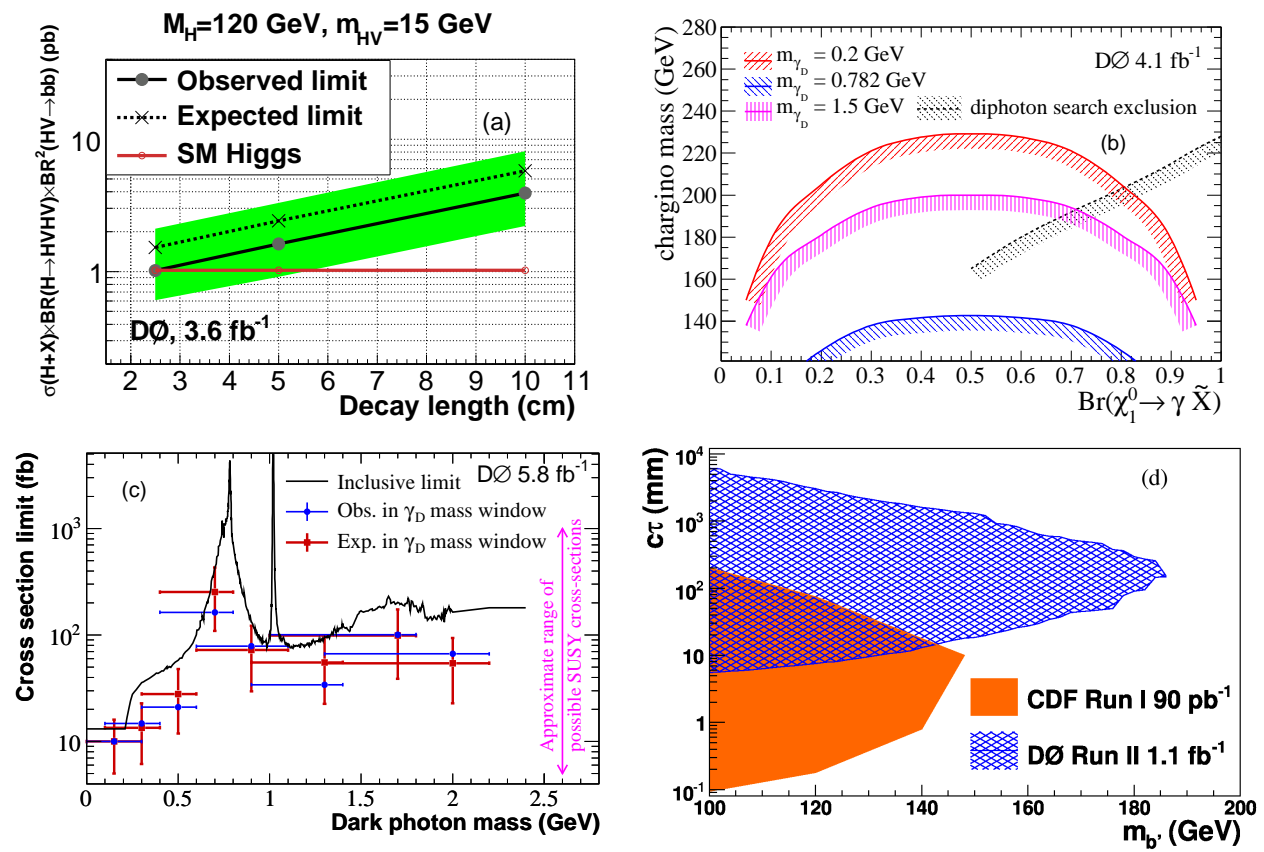

Fig. 16. (color online) Results from searches for the new particles from hidden-valley models from the D0 experiment. (a) The 95\% C.L. upper limits on the $\sigma \times \mathcal{B R}$ of the $H+X \rightarrow H V H V+X \rightarrow$ $b \bar{b} b \bar{b}+X$ as a function of the decay length. (b) The excluded region in the chargino mass vs. the $\mathcal{B R}$ of the $\tilde{\chi}_{1}^{0}$ into a photon for different dark photon masses from the search with a photon in final state. (c) The 95\% C.L. upper limit on $\sigma$ as a function on the dark photon mass in the search with leptonic-jets in final state. (d) The excluded region in the $c \tau_{b^{\prime}}$ vs. $m_{b^{\prime}}$ plane in the search for long-lived $b^{\prime} \rightarrow Z q \rightarrow e e q$.

\subsubsection{Charged massive stable particles}

Searches for CHAMPS are typically done by examining events for the presence of a single charged particle that behaves like a "heavy muon" in that it only interacts as a minimum ionizing particle as it traverses the detector. These particles can be produced directly (often as pairs) or as decay products of other particles. Both the $\mathrm{CDF}^{250}$ and the $\mathrm{D} \mathrm{0}^{251} 253$ experiments found no evidence for CHAMPS. Results are typically interpreted in SUSY models with limits as shown in Fig. 17(a,b) where the production mechanisms are gaugino-like, stop-like, or $\tilde{\tau}$-like CHAMPS. 

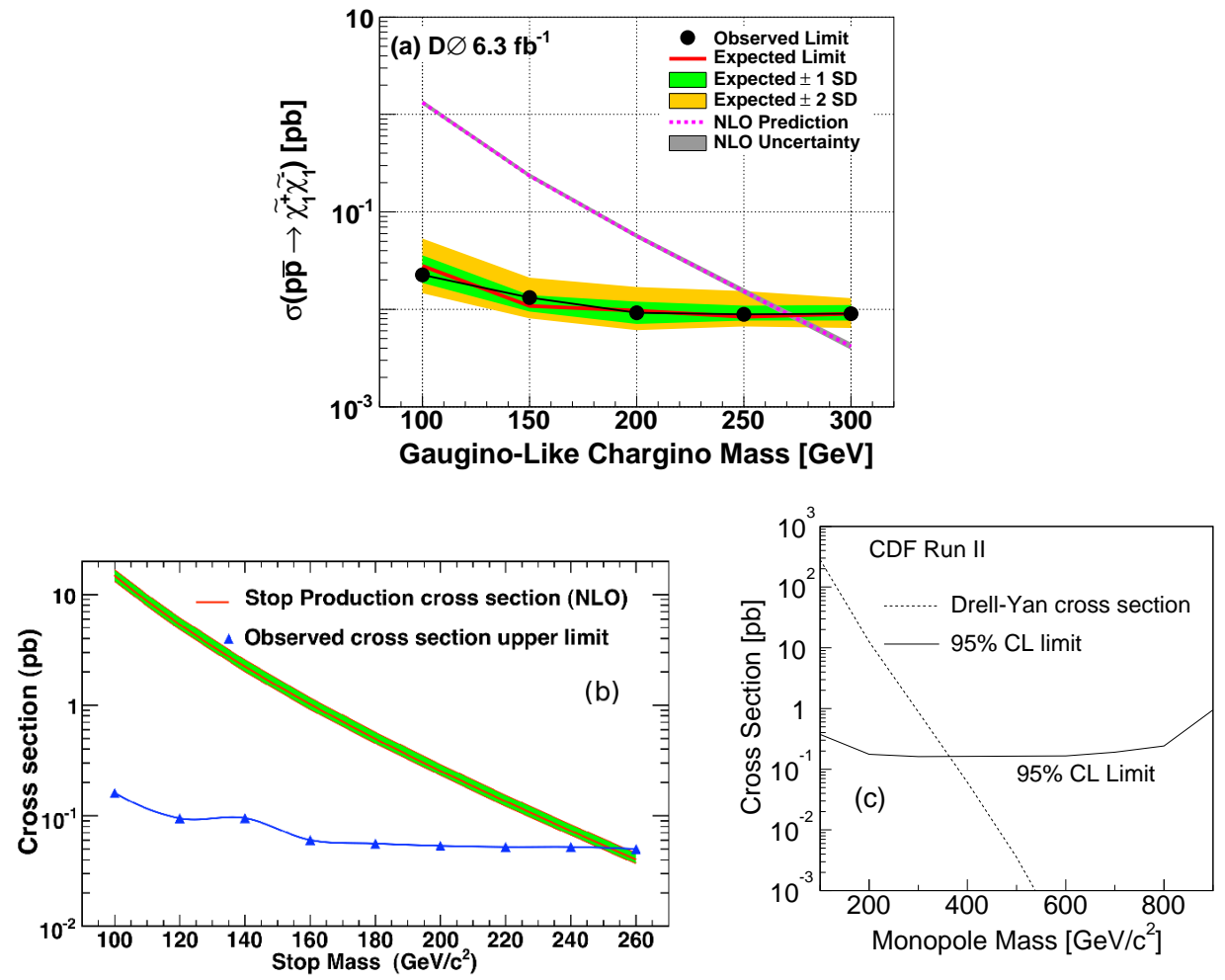

Fig. 17. (color online) Results for long-lived particle searches. The 95\% C.L. cross section upper limits for (a) gaugino-like charginos CHAMPS from the D0 experiment and for (b) top-squark CHAMPS from the CDF experiment. (c) The 95\% C.L. cross section upper limit as a function of magnetic monopole mass from the CDF experiment.

\subsubsection{Other searches for long-lived particles - monopoles, stopped gluinos and quirks}

Pairs of Dirac magnetic monopoles, if they exist in nature, are predicted to be directly produced in collisions. Because of their large mass and magnetic charge they will move differently through the magnetic field and can be identified by their late time-of-arrival at the outer parts of the detector. Searches for monopoles in Run II have been done at the CDF detector ${ }^{\sqrt{254}}$ with no evidence of new production. Monopoles, assuming simple models of Drell-Yan style production, are excluded at 95\% C.L. for masses smaller than $360 \mathrm{GeV}$ (see Fig 17(c)).

Searches for stopped gluinos are done by looking for $R$-hadrons that get trapped in the calorimeter. They can then decay up to 100 hours after their production. The search is done at the D0 experiment by looking for deposits of energy in the calorimeter which are not synchronized with an accelerator bunch crossing. Results ${ }^{255}$ are shown in the Fig. 18(a). 
The Fermilab neutrino experiment $\mathrm{NuTeV}$ observed an excess of dimuon events ${ }^{256}$ that could be interpreted as SUSY models with R-parity violation $\underline{8}$ or HV models ${ }^{[257}$ A follow-up analysis at the D0 experiment searched for pair-production of neutral particles each travelling for at least $5 \mathrm{~cm}$ before decaying into a pair of muons.258 No evidence is found and limits are set with results shown in the Fig. 18(b).

New particles known as quirks, $Q$ which are strongly interacting under their own $S U(N)$ force, can be pair produced at hadron colliders if they also carry SM charges. In addition to the quirk mass, the strength of the new $S U(N)$ gauge coupling, infracolor (which becomes strong at the scale $\Lambda$ ) is important phenomenologically. In a case when $\Lambda<<m_{Q} \simeq 0.1-1 \mathrm{TeV}$, breaking of the infracolor string is exponentially suppressed due to the large value of the ratio $m_{Q} / \Lambda$, and the quirkantiquirk pair stays connected by the infracolor string like a rubber band that can stretch to macroscopic length proportional to $m_{Q} / \Lambda^{2}$. The D0 experiment ${ }^{259}$ searched for cases where the extra gauge group is $S U(2), S U(3)$ or $S U(5)$. In these scenarios, we have the unusual signature that the individual quirks ionize atoms in the tracking chamber, but the macroscopic distance between the quirk and antiquark provide a neutral charged object that does not change direction as it traverses the detector. Thus, it can be reconstructed as a slow, highly ionizing, high $p_{T}$ track that decays after a few $\mathrm{cm}$. Since there could be a high $p_{T}$ jet from initial state radiation, the signature will consist of this special type of track, one jet, and large $E_{T}$ aligned with the track. No evidence for quirks are found and the results are shown in Fig. 18(c).

\subsection{Extra dimensions and dark matter}

While many models of extra dimensions are constrained by experiment, 9 there is significant room to allow the possibility of new particles and interactions. On the one hand many LED and UED models predict new particles, which will "leave" the detector without interacting, or they will interfere with SM processes in various final states; evidence for the models in former case would show up in ways that are similar to dark matter searches. On the other hand, excited KK modes of the graviton which are localized on the SM brane, spin-2 particles $G^{*}$, could produce resonances in ee, $\gamma \gamma, W W$ and $Z Z$ final states that are readily searched for.

The D0 and the CDF experiments searched for extra dimensions in a number of ways. We begin with a description of the searches for LED models in which Kaluza-Klein (KK) gravitons are directly produced but immediately disappear. In this case the gravitons are often produced with high transverse momentum and in association with a quark, a gluon, or a photon, giving rise to either monojet or monophoton final states with a large $E_{T}$ due to the escaping graviton. No evidence of new physics is observed $260 \sqrt{262}$ and results in the $M_{D}$ vs. $N_{D}$ plane, where $M_{D}$ the fundamental Planck scale in the $(4+n)$-dimensional space-time and $N_{D}$ is number of extra dimensions, are shown in Fig. 19(a). Other models indicate that evidence 

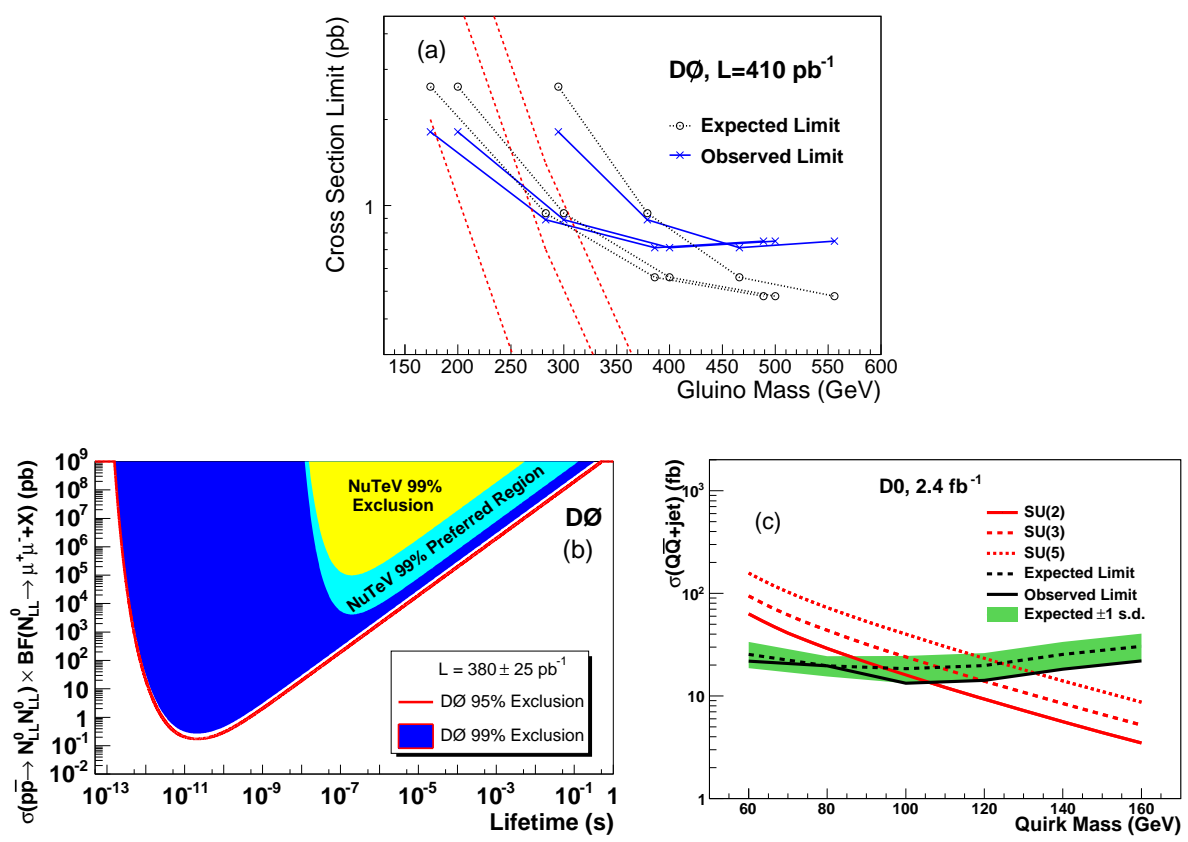

Fig. 18. (color online) (a) The 95\% C.L. cross section upper limits on stopped gluinos with the assumption that $\mathcal{B R}\left(\tilde{g} \rightarrow g \tilde{\chi}_{1}^{0}\right)=1$ from the D0 experiment. (b) The $95 \%$ C.L. upper limits on the $\sigma \times \mathcal{B R}$ of pair production of neutral long-lived particles decaying to pairs of muons as a function of their lifetime. (c) The 95\% C.L. cross section upper limits for pair of quirks and a jet (from initial state radiation) as a function of the mass of the quirk.

can be inferred in fermion and/or boson final states from the interference between

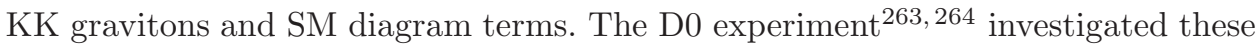
signatures in the $e e, \gamma \gamma$ and $\mu \mu$ final states by searching for deviations in the correlation between the invariant mass and the angular distribution of the pairs from SM-only predictions. Results from ee and $\gamma \gamma$ search are shown in Fig. 19(b).

The searches for UED processes typically focus on the production and decay of KK particles, denoted here with a * Typically production begins with KK gluons $\left(g^{*}\right)$ or quarks $\left(q^{*}\right)$ and decay via $q^{*} \rightarrow q Z^{*} \rightarrow q\left(l l^{*}\right) \rightarrow q l\left(l \gamma^{*}\right)$. In the case with only one extra dimension, minimal UED (mUED), the $\gamma^{*}$ is stable and is a dark matter candidate. This can result in a final state which includes two leptons (samesign or opposite sign) as well as a SM jet and $\mathbb{E}_{T}$. The D0 experiment ${ }^{265}$ searched for mUED in the same-sign lepton final state and excluded $R_{c}^{-1}$ up to $260 \mathrm{GeV}$, where $R_{c}$ is the radius of the compact dimension. This limit corresponds to a mass of $317 \mathrm{GeV}$ of the lightest KK quark. When additional extra dimensions exist the $\gamma^{*}$ decays via $\gamma^{*} \rightarrow \gamma+G$, where $G$ is graviton, yielding the $\gamma \gamma+E_{T}$ final state from pair production of $\gamma^{*}$. For the D0 search for UED, 177 a model with six extra dimensions, a fundamental Planck scale of $5 \mathrm{TeV}$, and $\mathcal{B R}\left(\gamma^{*} \rightarrow \gamma G\right) \approx 1$ yielded a 
limit of $R_{c}^{-1}<477 \mathrm{GeV}$ along with the other results shown in Fig. 19)(c).
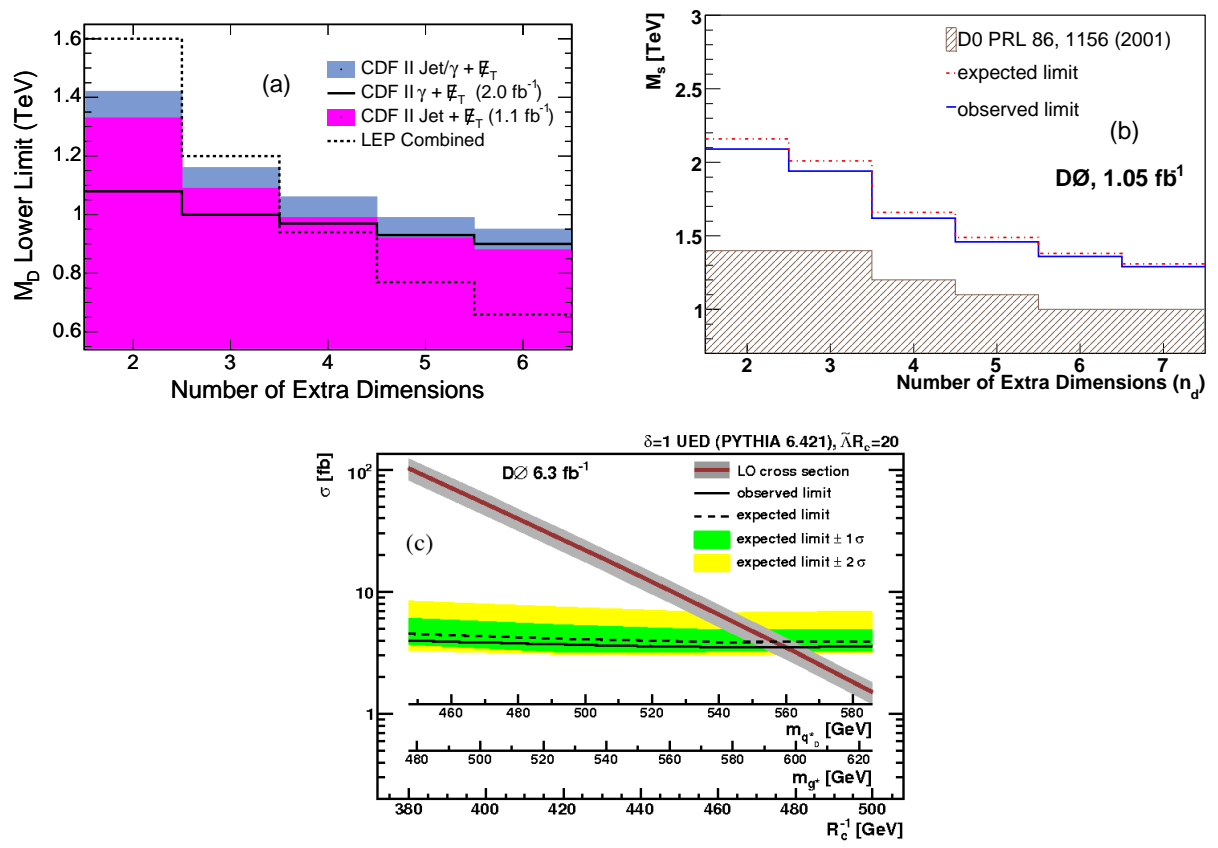

Fig. 19. (color online) Limits on extra dimensions from the CDF and D0 experiments. (a) The excluded region in the $M_{D}$ vs. $N_{D}$ plane in a search for LED from the combined monophoton and monojet final states from the CDF experiment. (b)The limits from the ee and $\gamma \gamma$ final states from the D0 experiment. (c) The $95 \%$ C.L. cross section upper limits for a UED model in the $\gamma \gamma+E_{T}$ final state from the D0 experiment.

Excited KK modes of the graviton, $G^{*}$, which are localized on the SM brane, are predicted in Randal-Sundrum (RS) models with a warped spacetime metric. Two parameters determine graviton couplings and widths: the constant $k / \bar{M}_{P L}$, where $k$ is the curvature scale of the extra dimension, and $\bar{M}_{P L}=M_{P L} / \sqrt{8}$ is the reduced Planck scale, and graviton excitation, $M_{1}$. The Do ${ }^{266+268}$ and the CDF experiments 211$] 269 \mid 270]$ searched for evidence of single RS graviton production and decay via $G^{*} \rightarrow \ell \ell$ or $V V$. No significant excess of events in the dilepton $(e$ or $\mu$ ) or $\gamma \gamma$ was found and limits are shown in Fig. 20(a,b). The results from the searches for the other diboson resonances described in the previous section were also interpreted as limits on the RS graviton production.216|217|219]271] Limits from the $G^{*} \rightarrow W W$ are set for a mass $m_{G^{*}}<754 \mathrm{GeV}$ when $k / \bar{M}_{P L}=0.1$. In $G^{*} \rightarrow Z Z$ an excess of events is observed in low yield four-lepton channel at the $M_{G^{*}}=327 \mathrm{GeV}$, but it was not confirmed in the more sensitive searches in the $\ell \ell j j$ and $\ell \ell E_{T}$ final states. 217 Figure 20(c) shows the results.

There are many ways to search for dark matter (DM) in high energy collisions 

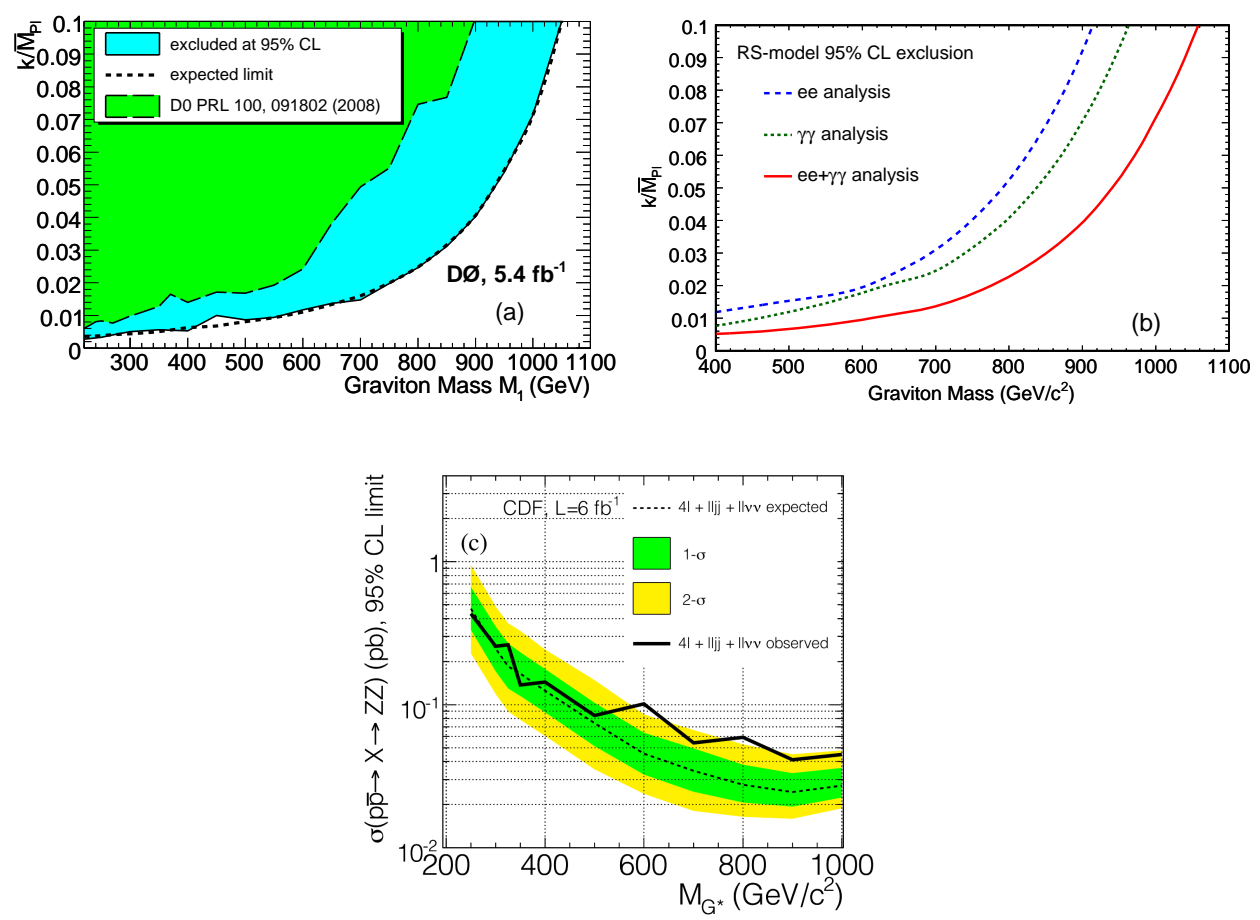

Fig. 20. (color online) The $95 \%$ C.L. excluded region in the $k / \bar{M}_{P L}$ vs. $M_{G}$ plane from a search for RS gravitons in the ee and $\gamma \gamma$ final states from (a) the D0 and (b) the CDF experiments. (c) The $95 \%$ C.L. upper limits on the cross section of the $G^{*} \rightarrow Z Z$ as a function of the $M_{G^{*}}$ from the CDF experiment.

depending on the potential production model. SUSY models, where the DM is the LSP and is produced in the cascade decays of other sparticles, were described in section 4. However, direct production is possible and can be observed if the DM particles are produced in association with a high energy photon or jet produced via initial state radiation. The process $p \bar{p} \rightarrow D M+D M+$ jet $\rightarrow$ jet $+E_{T}$ was investigated at the CDF experiment ${ }^{272}$ No significant deviations are found and the $90 \%$ C.L. cross section upper limits are set and converted into constraints on the DM-nucleon cross section. These results are shown together with several direct detection results in Fig. 21

\subsection{Signature-based searches and model-independent searches}

Following the early development of signature-based searches in Run I, as described in section 3.3 both the CDF and the D0 experiments did model-independent searches for new physics looking for discrepancies between data and SM predictions in the events characterized with high transverse momentum. These were done using 

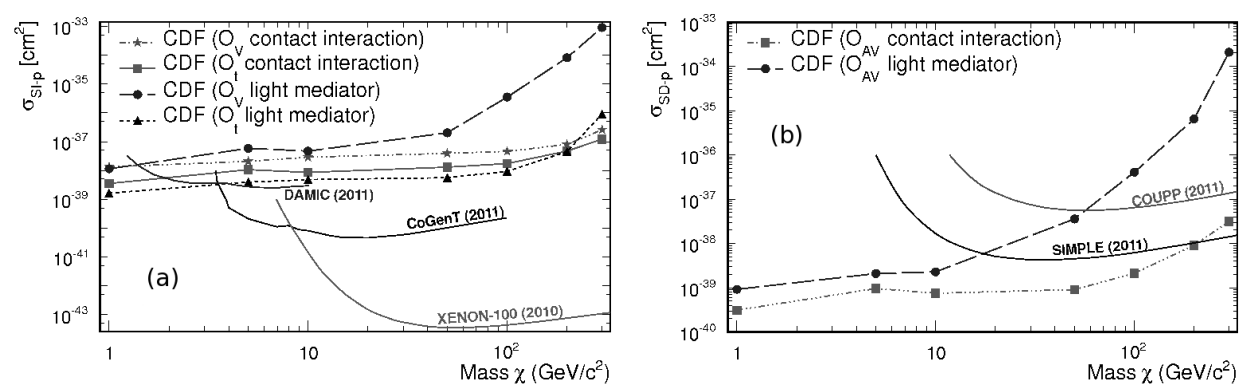

Fig. 21. (color online) The results on the DM-nucleon scattering from the CDF experiment, done at $90 \%$ C.L., compared to the other direct dark matter experiments. For a detailed description, see Ref. 272

the SLEUTH, BUMP HUNTER and VISTA programs 273 at the CDF experiment and similar methods at the D0 experiment ${ }^{275}$ Despite the huge number of final states considered, (SLEUTH considered 399 final states, BUMP HUNTER 5036 final states and VISTA considered 19650 final states), no true anomalies emerged (although the methods did serve to improve the MC simulation when discrepancies were noticed). The most discrepant final state contained $e \mathbb{E}_{T}+b$, but was found to be consistent when taking into account the trials factor. In addition, the CDF experiment searched for new physics in a number of dedicated signature-based searches, specifically: (i) $\gamma \gamma$, $\ell \gamma+E_{T}$ and $\ell \ell \gamma$ events, $276\left[277\right.$ where the famous ee $\gamma \gamma E_{T}$ event from Run I would

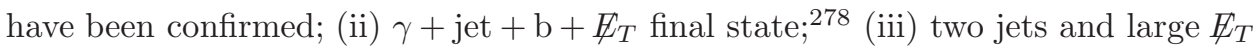
events, $\frac{279}{(i v) ~} Z Z+E_{T} \rightarrow \ell \ell q q+E_{T}$ events, $\frac{280}{2}$ and (v) $p \bar{p} \rightarrow(3 j$ jets $)(3 j e t s) \stackrel{2811}{.} \mathrm{In}$ all of these searches data agreed with the SM prediction, and no new physics was found.

\section{Summary and Conclusions}

The legacy of the Fermilab Tevatron collider experiments in searches for new particles and interactions is a powerful and glorious one. The searches for new particles, such as SUSY, new fermions and bosons, excited fermions, leptoquarks, technicolor, hidden-valley model particles, long-lived particles, extra dimensions, dark matter particles, and a host of other interesting signatures was broad and deep, and produced interesting hints that changed the way we look at searches today. Indeed many new theoretical models and experimental techniques came into favor because of the CDF and D0 experiments, and are followed closely by the LHC which has taken over the high energy frontier. In its time the answers from the Tevatron, both in terms of long-established models and new important ones that cropped up, quickly responded to the best ideas in the field and and provided inspiration for new theoretical ideas. 


\section{Acknowledgments}

We thank Ray Culbertson, Michael Eads, Paul Grannis, Oscar Gonzalez Lopez, and Stephen Mrenna for useful comments and discussions.

We thank the Fermilab staff and technical staffs of the participating institutions for their vital contributions. We acknowledge support from the DOE and NSF (USA), ARC (Australia), CNPq, FAPERJ, FAPESP and FUNDUNESP (Brazil), NSERC (Canada), NSC, CAS and CNSF (China), Colciencias (Colombia), MSMT and GACR (Czech Republic), the Academy of Finland, CEA and CNRS/IN2P3 (France), BMBF and DFG (Germany), DAE and DST (India), SFI (Ireland), INFN (Italy), MEXT (Japan), the KoreanWorld Class University Program and NRF (Korea), CONACyT (Mexico), FOM (Netherlands), MON, NRC KI and RFBR (Russia), the Slovak R\&D Agency, the Ministerio de Ciencia e Innovacion, and Programa Consolider-Ingenio 2010 (Spain), The Swedish Research Council (Sweden), SNSF (Switzerland), STFC and the Royal Society (United Kingdom), the A.P. Sloan Foundation (USA), and the EU community Marie Curie Fellowship contract 302103.

One author (L. Z.) is supported by Serbian Ministry of Education, Science and Technological development project 171004 . One author (D. T.) is supported by the Mitchell Institute of Fundamental Physics and Astronomy. 


\section{References}

1. M. Baak et al., Eur. Phys. J. C 72, 2205 (2012).

2. ATLAS Collaboration (G. Aad et al.), Phys. Lett. B 716, 1 (2012).

3. CMS Collaboration (S. Chatrchyan et al.), Phys. Lett. B 716, 30 (2012).

4. J. Wess and B. Zumino, Nucl. Phys. B 70, 39 (1974).

5. P. Fayet and S. Ferrara, Phys. Rept. 32, 249 (1977).

6. H. P. Nilles, Phys.Rept. 110, 1 (1984).

7. H. E. Haber and G. L. Kane, Phys.Rept. 117, 75 (1985).

8. S. P. Martin, arXiv:hep-ph/9709356 (1997).

9. Particle Data Group (J. Beringer et al.), Phys. Rev. D 86, 010001 (2012) and 2013 partial update for the 2014 edition.

10. LEP Collaboration, ALEPH Collaboration, DELPHI Collaboration, L3 Collaboration, OPAL Collaboration, Line Shape Sub-Group of the LEP Electroweak Working Group (R. Barate et al.), arXiv:hep-ex/0101027 (2001).

11. G. D. Kribs, T. Plehn, M. Spannowsky and T. M. P. Tait, Phys. Rev. D 76, 075016 (2007).

12. P. H. Frampton, P. Hung and M. Sher, Phys. Rept. 330, 263 (2000).

13. A. Atre, M. Carena, T. Han and J. Santiago, Phys. Rev. D 79, 054018 (2009).

14. R. N. Mohapatra and J. C. Pati, Phys. Rev. D 11, 566 (1975).

15. R. Mohapatra and J. C. Pati, Phys. Rev. D 11, 2558 (1975).

16. G. Senjanovic and R. N. Mohapatra, Phys. Rev. D 12, 1502 (1975).

17. P. Langacker, Rev. Mod. Phys. 81, 1199 (2009).

18. U. Baur, M. Spira and P. Zerwas, Phys. Rev. D 42, 815 (1990).

19. J. H. Schwarz, arXiv:hep-ex/0008017 (2001).

20. W. Buchmuller, R. Ruckl and D. Wyler, Phys. Lett. B 191, 442 (1987), Erratum-ibid. B 448320 (1999).

21. S. Weinberg, Phys. Rev. D 19, 1277 (1979).

22. L. Susskind, Phys. Rev. D 20, 2619 (1979).

23. C. T. Hill and E. H. Simmons, Phys. Rept. 381, 235 (2003).

24. K. Lane and S. Mrenna, Phys. Rev. D 67, 115011 (2003).

25. CDF Collaboration (T. Aaltonen et al.), Phys.Rev.Lett. 101, 202001 (2008).

26. D0 Collaboration (V. Abazov et al.), Phys.Rev.Lett. 100, 142002 (2008).

27. CDF Collaboration (T. Aaltonen et al.), Phys. Rev. D 83, 112003 (2011).

28. C. E. Gerber and C. Vellidis, Review of Tevatron Results: Top quark physics (2014), arXiv: xxx [hep-ex], Submitted to Int. J. Mod. Phys. A.

29. CDF Collaboration (T. Aaltonen et al.), Phys. Rev. Lett. 106, 171801 (2011).

30. T. Han, Z. Si, K. M. Zurek and M. J. Strassler, J. High Energy Phys. 0807, 008 (2008).

31. N. Arkani-Hamed, D. P. Finkbeiner, T. R. Slatyer and N. Weiner, Phys. Rev. D 79, 015014 (2009).

32. M. Baumgart, C. Cheung, J. T. Ruderman, L.-T. Wang and I. Yavin, J. High Energy Phys. 0904, 014 (2009).

33. M. Byrne, C. Kolda and P. Regan, Phys. Rev. D 66, 075007 (2002).

34. J. Kang and M. A. Luty, J. High Energy Phys. 0911, 065 (2009).

35. N. Arkani-Hamed, S. Dimopoulos and G. Dvali, Phys. Lett. B 429, 263 (1998).

36. T. Appelquist, H.-C. Cheng and B. A. Dobrescu, Phys. Rev. D 64, 035002 (2001).

37. L. Randall and R. Sundrum, Phys. Rev. Lett. 83, 3370 (1999).

38. G. Bertone, D. Hooper and J. Silk, Phys. Rept. 405, 279 (2005).

39. J. L. Feng, Ann. Rev. Astron. Astrophys. 48, 495 (2010).

40. L. D. Duffy and K. van Bibber, New J. Phys. 11, 105008 (2009). 
41. CDF Collaboration (F. Abe et al.), Phys. Rev. Lett. 63, 1447 (1989).

42. CDF Collaboration (F. Abe et al.), Phys. Rev. Lett. 62, 1825 (1989).

43. CDF Collaboration (F. Abe et al.), Phys. Rev. Lett. 64, 147 (1990).

44. CDF Collaboration (F. Abe et al.), Phys. Rev. Lett. 67, 2609 (1991).

45. CDF Collaboration (F. Abe et al.), Phys. Rev. D 46, R1889 (1992).

46. CDF Collaboration (F. Abe et al.), Phys. Rev. Lett. 69, 3439 (1992).

47. CDF Collaboration (F. Abe et al.), Phys. Rev. Lett. 68, 1463 (1992).

48. CDF Collaboration (F. Abe et al.), Phys. Rev. D 48, R3939 (1993).

49. CDF Collaboration (F. Abe et al.), Phys. Rev. Lett. 71, 2542 (1993).

50. CDF Collaboration (F. Abe et al.), Phys. Rev. Lett. 72, 3004 (1994).

51. CDF Collaboration (F. Abe et al.), Phys. Rev. Lett. 74, 3538 (1995).

52. CDF Collaboration (F. Abe et al.), Phys. Rev. D 51, R949 (1995).

53. CDF Collaboration (F. Abe et al.), Phys. Rev. Lett. 75, 1012 (1995).

54. CDF Collaboration (F. Abe et al.), Phys. Rev. Lett. 75, 613 (1995).

55. CDF Collaboration (F. Abe et al.), Phys. Rev. Lett. 74, 2900 (1995).

56. CDF Collaboration (F. Abe et al.), Phys. Rev. Lett. 76, 2006 (1996).

57. CDF Collaboration (F. Abe et al.), Phys. Rev. Lett. 76, 4307 (1996).

58. CDF Collaboration (F. Abe et al.), Phys. Rev. Lett. 78, 2906 (1997).

59. CDF Collaboration (F. Abe et al.), Phys. Rev. D 55, R5263 (1997).

60. CDF Collaboration (F. Abe et al.), Phys. Rev. Lett. 80, 5275 (1998).

61. CDF Collaboration (F. Abe et al.), Phys. Rev. D 58, 051102 (1998).

62. CDF Collaboration (F. Abe et al.), Phys. Rev. Lett. 81, 1791 (1998).

63. CDF Collaboration (F. Abe et al.), Phys. Rev. Lett. 81, 4806 (1998).

64. CDF Collaboration (F. Abe et al.), Phys. Rev. Lett. 82, 2038 (1999).

65. CDF Collaboration (F. Abe et al.), Phys. Rev. D 59, 092002 (1999).

66. CDF Collaboration (F. Abe et al.), Phys. Rev. Lett. 82, 3206 (1999).

67. CDF Collaboration (F. Abe et al.), Phys. Rev. Lett. 83, 2133 (1999).

68. CDF Collaboration (F. Abe et al.), Phys. Rev. Lett. 83, 3124 (1999).

69. CDF Collaboration (T. Affolder et al.), Phys. Rev. Lett. 84, 5704 (2000).

70. CDF Collaboration (T. Affolder et al.), Phys. Rev. Lett. 84, 1110 (2000).

71. CDF Collaboration (T. Affolder et al.), Phys. Rev. Lett. 84, 835 (2000).

72. CDF Collaboration (F. Abe et al.), Phys. Rev. Lett. 84, 5716 (2000).

73. CDF Collaboration (T. Affolder et al.), Phys. Rev. Lett. 84, 5273 (2000).

74. CDF Collaboration (T. Affolder et al.), Phys. Rev. Lett. 85, 2062 (2000).

75. CDF Collaboration (T. Affolder et al.), Phys. Rev. Lett. 85, 1378 (2000).

76. CDF Collaboration (T. Affolder et al.), Phys. Rev. Lett. 85, 2056 (2000).

77. CDF Collaboration (T. Affolder et al.), Phys. Rev. D 63, 091101 (2001).

78. CDF Collaboration (T. Affolder et al.), Phys. Rev. D 64, 092002 (2001).

79. CDF Collaboration (T. Affolder et al.), Phys. Rev. Lett. 87, 231803 (2001).

80. CDF Collaboration (T. Affolder et al.), Phys. Rev. Lett. 88, 041801 (2002).

81. CDF Collaboration (T. Affolder et al.), Phys. Rev. Lett. 87, 251803 (2001).

82. CDF Collaboration (T. Affolder et al.), Phys. Rev. D 65, 052006 (2002).

83. CDF Collaboration (T. Affolder et al.), Phys. Rev. Lett. 88, 071806 (2002).

84. CDF Collaboration (D. Acosta et al.), Phys. Rev. D 66, 012004 (2002).

85. CDF Collaboration (D. Acosta et al.), Phys. Rev. Lett. 89, 041802 (2002).

86. CDF Collaboration (D. Acosta et al.), Phys. Rev. Lett. 89, 281801 (2002).

87. CDF Collaboration (D. Acosta et al.), Phys. Rev. Lett. 90, 131801 (2003).

88. CDF Collaboration (D. Acosta et al.), Phys. Rev. Lett. 90, 251801 (2003).

89. CDF Collaboration (D. Acosta et al.), Phys. Rev. Lett. 91, 171602 (2003).

90. CDF Collaboration (D. Acosta et al.), Phys. Rev. Lett. 92, 051803 (2004). 
91. CDF Collaboration (D. Acosta et al.), Phys. Rev. Lett. 92, 121802 (2004).

92. CDF Collaboration (D. Acosta et al.), Phys. Rev. Lett. 93, 061802 (2004).

93. D0 Collaboration (S. Abachi et al.), Phys. Rev. Lett. 75, 618 (1995).

94. D0 Collaboration (S. Abachi et al.), Phys. Lett. B 358, 405 (1995).

95. D0 Collaboration (S. Abachi et al.), Phys. Rev. Lett. 76, 2228 (1996).

96. D0 Collaboration (S. Abachi et al.), Phys. Rev. Lett. 76, 2222 (1996).

97. D0 Collaboration (S. Abachi et al.), Phys. Rev. Lett. 76, 3271 (1996).

98. D0 Collaboration (S. Abachi et al.), Phys. Lett. B 385, 471 (1996).

99. D0 Collaboration (S. Abachi et al.), Phys. Rev. Lett. 78, 2070 (1997).

100. D0 Collaboration (S. Abachi et al.), Phys. Rev. Lett. 78, 3818 (1997).

101. D0 Collaboration (B. Abbott et al.), Phys. Rev. Lett. 80, 442 (1998).

102. D0 Collaboration (B. Abbott et al.), Phys. Rev. Lett. 80, 1591 (1998).

103. D0 Collaboration (S. Abachi et al.), Phys. Rev. D 57, 589 (1998).

104. D0 Collaboration (B. Abbott et al.), Phys. Rev. Lett. 80, 666 (1998).

105. D0 Collaboration (B. Abbott et al.), Phys. Rev. Lett. 81, 524 (1998).

106. D0 Collaboration (B. Abbott et al.), Phys. Rev. Lett. 82, 29 (1999).

107. D0 Collaboration (B. Abbott et al.), Phys. Rev. Lett. 83, 4937 (1999).

108. D0 Collaboration (B. Abbott et al.), Phys. Rev. D 60, 031101 (1999).

109. D0 Collaboration (B. Abbott et al.), Phys. Rev. Lett. 83, 4476 (1999).

110. D0 Collaboration (B. Abbott et al.), Phys. Rev. Lett. 82, 2457 (1999).

111. D0 Collaboration (B. Abbott et al.), Phys. Rev. Lett. 82, 4769 (1999).

112. D0 Collaboration (B. Abbott et al.), Phys. Rev. D 62, 071701 (2000).

113. D0 Collaboration (B. Abbott et al.), Phys. Rev. D 62, 031101 (2000).

114. D0 Collaboration (B. Abbott et al.), Phys. Rev. D 62, 092004 (2000).

115. D0 Collaboration (B. Abbott et al.), Phys. Rev. D 63, 091102 (2001).

116. D0 Collaboration (B. Abbott et al.), Phys. Rev. Lett. 86, 1156 (2001).

117. D0 Collaboration (V. M. Abazov et al.), Phys. Rev. Lett. 87, 061802 (2001).

118. D0 Collaboration (V. M. Abazov et al.), Phys. Rev. D 64, 012004 (2001).

119. D0 Collaboration (V. M. Abazov et al.), Phys. Rev. Lett. 87, 231801 (2001).

120. D0 Collaboration (V. M. Abazov et al.), Phys. Rev. Lett. 88, 171802 (2002).

121. D0 Collaboration (V. M. Abazov et al.), Phys. Rev. Lett. 89, 171801 (2002).

122. D0 Collaboration (V. M. Abazov et al.), Phys. Rev. Lett. 89, 261801 (2002).

123. D0 Collaboration (V. M. Abazov et al.), Phys. Rev. D 66, 112001 (2002).

124. D0 Collaboration (V. M. Abazov et al.), Phys. Rev. Lett. 90, 251802 (2003).

125. D0 Collaboration (V. Abazov et al.), Phys. Lett. B 581, 147 (2004).

126. D0 Collaboration (V. M. Abazov et al.), Phys. Rev. Lett. 93, 011801 (2004).

127. D0 Collaboration (V. M. Abazov et al.), Phys. Rev. Lett. 92, 221801 (2004).

128. D0 Collaboration (V. Abazov et al.), Phys. Rev. D 69, 111101 (2004).

129. H1 Collaboration (C. Adloff et al.), Z. Phys. C 74, 191 (1997).

130. ZEUS Collaboration (J. Breitweg et al.), Z. Phys. C 74, 207 (1997).

131. D0 Collaboration (S. Abachi et al.), Phys. Rev. Lett. 72, 965 (1994).

132. D0 Collaboration (S. Abachi et al.), Phys. Rev. Lett. 75, 3618 (1995).

133. CDF Collaboration (F. Abe et al.), Phys. Rev. Lett. 79, 4327 (1997).

134. D0 Collaboration (B. Abbott et al.), Phys. Rev. Lett. 79, 4321 (1997).

135. D0 Collaboration (B. Abbott et al.), Phys. Rev. Lett. 80, 2051 (1998).

136. D0 Collaboration (V. M. Abazov et al.), Phys. Rev. D 64, 092004 (2001).

137. D0 Collaboration (B. Abbott et al.), Phys. Rev. Lett. 83, 2896 (1999).

138. D0 Collaboration (B. Abbott et al.), Phys. Rev. Lett. 84, 2088 (2000).

139. D0 Collaboration (B. Abbott et al.), Phys. Rev. Lett. 81, 38 (1998).

140. CDF Collaboration (F. Abe et al.), Phys. Rev. Lett. 81, 5742 (1998). 
141. D0 Collaboration (V. M. Abazov et al.), Phys. Rev. Lett. 88, 191801 (2002).

142. D0 Collaboration (B. Abbott et al.), Phys. Rev. Lett. 86, 3712 (2001).

143. H1 Collaboration (A. Aktas et al.), Phys. Lett. B 602, 14 (2004).

144. J. Lewis and R. V. Kooten, Review of Tevatron Results: Heavy Flavor Physics (2014), arXiv: xxx [hep-ex], Submitted to Int. J. Mod. Phys. A.

145. CDF Collaboration (T. Aaltonen et al.), Phys. Rev. Lett. 102, 121801 (2009).

146. D0 Collaboration (V. Abazov et al.), Phys. Lett. B 638, 119 (2006).

147. D0 Collaboration (V. Abazov et al.), Phys. Lett. B 660, 449 (2008).

148. D0 Collaboration (V. Abazov et al.), Phys. Lett. B 680, 24 (2009).

149. CDF Collaboration (T. Aaltonen et al.), Phys. Rev. Lett. 105, 081802 (2010).

150. D0 Collaboration (V. M. Abazov et al.), Phys. Rev. Lett. 97, 171806 (2006).

151. D0 Collaboration (V. M. Abazov et al.), Phys. Lett. B 693, 95 (2010).

152. CDF Collaboration (A. Abulencia et al.), Phys. Rev. Lett. 96, 171802 (2006).

153. CDF Collaboration (T. Aaltonen et al.), Phys. Rev. Lett. 102, 221801 (2009).

154. CDF Collaboration (T. Aaltonen et al.), Phys. Rev. D 82, 092001 (2010).

155. D0 Collaboration (V. Abazov et al.), Phys. Lett. B 659, 500 (2008).

156. D0 Collaboration (V. Abazov et al.), Phys. Lett. B 675, 289 (2009).

157. D0 Collaboration (V. M. Abazov et al.), Phys. Lett. B 696, 321 (2011).

158. D0 Collaboration (V. M. Abazov et al.), Phys. Lett. B 710, 578 (2012).

159. CDF Collaboration (T. Aaltonen et al.), Phys. Rev. Lett. 104, 251801 (2010).

160. D0 Collaboration (V. Abazov et al.), Phys. Lett. B 674, 4 (2009).

161. CDF Collaboration (T. Aaltonen et al.), J. High Energy Phys. 1210, 158 (2012).

162. CDF Collaboration (T. Aaltonen et al.), Phys. Rev. D 76, 072010 (2007).

163. D0 Collaboration (V. Abazov et al.), Phys. Lett. B 645, 119 (2007).

164. D0 Collaboration (V. Abazov et al.), Phys. Lett. B 665, 1 (2008).

165. CDF Collaboration (T. Aaltonen et al.), Phys. Rev. Lett. 101, 251801 (2008).

166. CDF Collaboration (T. A. Aaltonen et al.), Phys. Rev. D 90, 012011 (2014).

167. D0 Collaboration (V. Abazov et al.), Phys. Lett. B 680, 34 (2009).

168. D0 Collaboration (V. M. Abazov et al.), Phys. Rev. Lett. 95, 151805 (2005).

169. CDF Collaboration (A. Abulencia et al.), Phys. Rev. Lett. 98, 221803 (2007).

170. CDF Collaboration (T. Aaltonen et al.), Phys. Rev. Lett. 110, 201802 (2013).

171. B. Allanach et al., Eur. Phys. J. C 25, 113 (2002).

172. D. Toback and P. Wagner, Phys. Rev. D 70, 114032 (2004).

173. CDF Collaboration (D. Acosta et al.), Phys. Rev. D 71, 031104 (2005).

174. CDF Collaboration (T. Aaltonen et al.), Phys. Rev. Lett. 104, 011801 (2010).

175. D0 Collaboration (V. Abazov et al.), Phys. Lett. B 659, 856 (2008).

176. D0 Collaboration (V. M. Abazov et al.), Phys. Rev. Lett. 94, 041801 (2005).

177. D0 Collaboration (V. M. Abazov et al.), Phys. Rev. Lett. 105, 221802 (2010).

178. M. Goncharov et al., Nucl. Instrum. Methods Phys. Res. A 565, 543 (2006).

179. CDF Collaboration (T. Aaltonen et al.), Phys. Rev. D 78, 032015 (2008).

180. CDF Collaboration (A. Abulencia et al.), Phys. Rev. Lett. 99, 121801 (2007).

181. D0 Collaboration (V. M. Abazov et al.), Phys. Rev. D 86, 071701 (2012).

182. J. D. Mason and D. Toback, Phys. Lett. B 702, 377 (2011).

183. CDF Collaboration (T. Aaltonen et al.), Phys. Rev. D 88, 031103 (2013).

184. CDF Collaboration (T. Aaltonen et al.), Phys. Rev. Lett. 101, 071802 (2008).

185. CDF Collaboration (A. Abulencia et al.), Phys. Rev. Lett. 96, 211802 (2006).

186. CDF Collaboration (T. Aaltonen et al.), Phys. Rev. Lett. 105, 191801 (2010).

187. D0 Collaboration (V. M. Abazov et al.), Phys. Rev. Lett. 100, 241803 (2008).

188. D0 Collaboration (V. M. Abazov et al.), Phys. Rev. Lett. 105, 191802 (2010).

189. D0 Collaboration (V. Abazov et al.), Phys. Lett. B 638, 441 (2006). 
190. D0 Collaboration (V. M. Abazov et al.), Phys. Rev. Lett. 97, 111801 (2006).

191. T. R. Junk and A. Juste, Review of Tevatron Results: Higgs Boson Physics (2014), arXiv: xxx [hep-ex], Submitted to Int. J. Mod. Phys. A.

192. CDF Collaboration (T. Aaltonen et al.), Phys. Rev. D 76, 072006 (2007).

193. D0 Collaboration (V. M. Abazov et al.), Phys. Rev. Lett. 106, 081801 (2011).

194. CDF Collaboration (T. Aaltonen et al.), Phys. Rev. Lett. 104, 091801 (2010).

195. CDF Collaboration (T. Aaltonen et al.), Phys. Rev. Lett. 106, 141803 (2011).

196. D0 Collaboration (V. M. Abazov et al.), Phys. Rev. Lett. 107, 082001 (2011).

197. CDF Collaboration (T. Aaltonen et al.), Phys. Rev. Lett. 108, 211805 (2012).

198. CDF Collaboration (T. Aaltonen et al.), Phys. Rev. Lett. 107, 261801 (2011).

199. CDF Collaboration (T. Aaltonen et al.), Phys. Rev. Lett. 106, 191801 (2011).

200. CDF Collaboration (T. Aaltonen et al.), Phys. Rev. Lett. 100, 161803 (2008).

201. CDF Collaboration (A. Abulencia et al.), Phys. Rev. D 75, 091101 (2007).

202. D0 Collaboration (V. M. Abazov et al.), Phys. Rev. Lett. 100, 031804 (2008).

203. CDF Collaboration (T. Aaltonen et al.), Phys. Rev. D 83, 031102 (2011).

204. CDF Collaboration (T. Aaltonen et al.), Phys. Rev. Lett. 103, 041801 (2009).

205. D0 Collaboration (V. Abazov et al.), Phys. Lett. B 641, 423 (2006).

206. D0 Collaboration (V. M. Abazov et al.), Phys. Rev. Lett. 100, 211803 (2008).

207. D0 Collaboration (V. M. Abazov et al.), Phys. Lett. B 699, 145 (2011).

208. D0 Collaboration (V. M. Abazov et al.), Phys. Lett. B 695, 88 (2011).

209. CDF Collaboration (T. Aaltonen et al.), Phys. Rev. Lett. 106, 121801 (2011).

210. CDF Collaboration (T. Aaltonen et al.), Phys. Rev. Lett. 102, 091805 (2009).

211. CDF Collaboration (T. Aaltonen et al.), Phys. Rev. Lett. 102, 031801 (2009).

212. CDF Collaboration (T. Aaltonen et al.), Phys. Rev. D 79, 112002 (2009).

213. CDF Collaboration (T. Aaltonen et al.), Phys. Rev. Lett. 110, 121802 (2013).

214. D0 Collaboration (V. Abazov et al.), Phys. Lett. B 668, 98 (2008).

215. D0 Collaboration (V. M. Abazov et al.), Phys. Rev. D 85, 051101 (2012).

216. CDF Collaboration (T. Aaltonen et al.), Phys. Rev. Lett. 104, 241801 (2010).

217. CDF Collaboration (T. Aaltonen et al.), Phys. Rev. D 85, 012008 (2012).

218. D0 Collaboration (V. M. Abazov et al.), Phys. Rev. Lett. 104, 061801 (2010).

219. D0 Collaboration (V. M. Abazov et al.), Phys. Rev. Lett. 107, 011801 (2011).

220. CDF Collaboration (T. Aaltonen et al.), Phys. Rev. Lett. 111, 031802 (2013).

221. D0 Collaboration (V. M. Abazov et al.), Phys. Rev. D 77, 091102 (2008).

222. CDF Collaboration (D. Acosta et al.), Phys. Rev. Lett. 94, 101802 (2005).

223. CDF Collaboration (A. Abulencia et al.), Phys. Rev. Lett. 97, 191802 (2006).

224. D0 Collaboration (V. M. Abazov et al.), Phys. Rev. D 73, 111102 (2006).

225. D0 Collaboration (V. M. Abazov et al.), Phys. Rev. D 74, 011104 (2006).

226. CDF Collaboration (T. Aaltonen et al.), Phys. Rev. D 77, 091105 (2008).

227. CDF Collaboration (A. Abulencia et al.), Phys. Rev. D 73, 051102 (2006).

228. CDF Collaboration (D. Acosta et al.), Phys. Rev. D 72, 051107 (2005).

229. CDF Collaboration (D. Acosta et al.), Phys. Rev. D 71, 112001 (2005), Erratum-ibid. D 71119901 (2005).

230. D0 Collaboration (V. M. Abazov et al.), Phys. Rev. D 71, 071104 (2005).

231. D0 Collaboration (V. Abazov et al.), Phys. Lett. B 681, 224 (2009).

232. D0 Collaboration (V. M. Abazov et al.), Phys. Rev. D 84, 071104 (2011).

233. D0 Collaboration (V. Abazov et al.), Phys. Lett. B 636, 183 (2006).

234. D0 Collaboration (V. Abazov et al.), Phys. Lett. B 647, 74 (2007).

235. D0 Collaboration (V. Abazov et al.), Phys. Lett. B 671, 224 (2009).

236. D0 Collaboration (V. M. Abazov et al.), Phys. Rev. Lett. 99, 061801 (2007).

237. D0 Collaboration (V. M. Abazov et al.), Phys. Rev. Lett. 101, 241802 (2008). 
238. D0 Collaboration (V. Abazov et al.), Phys. Lett. B 640, 230 (2006).

239. D0 Collaboration (V. Abazov et al.), Phys. Lett. B 668, 357 (2008).

240. D0 Collaboration (V. M. Abazov et al.), Phys. Rev. Lett. 98, 221801 (2007).

241. CDF Collaboration (T. Aaltonen et al.), Phys. Rev. Lett. 104, 111802 (2010).

242. CDF Collaboration (T. Aaltonen et al.), Phys. Rev. Lett. 112, 111803 (2014).

243. D0 Collaboration (V. M. Abazov et al.), Phys. Rev. Lett. 107, 011804 (2011).

244. CDF Collaboration (T. Aaltonen et al.), Phys. Rev. D 88, 092004 (2013).

245. CDF Collaboration (T. Aaltonen et al.), Phys. Rev. D 89, 092001 (2014).

246. D0 Collaboration (V. M. Abazov et al.), Phys. Rev. Lett. 103, 071801 (2009).

247. D0 Collaboration (V. M. Abazov et al.), Phys. Rev. Lett. 103, 081802 (2009).

248. D0 Collaboration (V. M. Abazov et al.), Phys. Rev. Lett. 105, 211802 (2010).

249. D0 Collaboration (V. M. Abazov et al.), Phys. Rev. Lett. 101, 111802 (2008).

250. CDF Collaboration (T. Aaltonen et al.), Phys. Rev. Lett. 103, 021802 (2009).

251. D0 Collaboration (V. M. Abazov et al.), Phys. Rev. Lett. 102, 161802 (2009).

252. D0 Collaboration (V. M. Abazov et al.), Phys. Rev. Lett. 108, 121802 (2012).

253. D0 Collaboration (V. M. Abazov et al.), Phys. Rev. D 87, 052011 (2013).

254. CDF Collaboration (A. Abulencia et al.), Phys. Rev. Lett. 96, 201801 (2006).

255. D0 Collaboration (V. M. Abazov et al.), Phys. Rev. Lett. 99, 131801 (2007).

256. NuTeV Collaboration (T. Adams et al.), Phys. Rev. Lett. 87, 041801 (2001).

257. M. J. Strassler and K. M. Zurek, Phys. Lett. B 651, 374 (2007).

258. D0 Collaboration (V. M. Abazov et al.), Phys. Rev. Lett. 97, 161802 (2006).

259. D0 Collaboration (V. M. Abazov et al.), Phys. Rev. Lett. 105, 211803 (2010).

260. D0 Collaboration (V. M. Abazov et al.), Phys. Rev. Lett. 101, 011601 (2008).

261. CDF Collaboration (T. Aaltonen et al.), Phys. Rev. Lett. 101, 181602 (2008).

262. CDF Collaboration (A. Abulencia et al.), Phys. Rev. Lett. 97, 171802 (2006).

263. D0 Collaboration (V. M. Abazov et al.), Phys. Rev. Lett. 102, 051601 (2009).

264. D0 Collaboration (V. M. Abazov et al.), Phys. Rev. Lett. 95, 161602 (2005).

265. D0 Collaboration (V. M. Abazov et al.), Phys. Rev. Lett. 108, 131802 (2012).

266. D0 Collaboration (V. M. Abazov et al.), Phys. Rev. Lett. 95, 091801 (2005).

267. D0 Collaboration (V. M. Abazov et al.), Phys. Rev. Lett. 100, 091802 (2008).

268. D0 Collaboration (V. M. Abazov et al.), Phys. Rev. Lett. 104, 241802 (2010).

269. CDF Collaboration (T. Aaltonen et al.), Phys. Rev. Lett. 107, 051801 (2011).

270. CDF Collaboration (T. Aaltonen et al.), Phys. Rev. D 83, 011102 (2011).

271. CDF Collaboration (T. Aaltonen et al.), Phys. Rev. D 83, 112008 (2011).

272. CDF Collaboration (T. Aaltonen et al.), Phys. Rev. Lett. 108, 211804 (2012).

273. CDF Collaboration (T. Aaltonen et al.), Phys. Rev. D 78, 012002 (2008).

274. CDF Collaboration (T. Aaltonen et al.), Phys. Rev. D 79, 011101 (2009).

275. D0 Collaboration (V. M. Abazov et al.), Phys. Rev. D 85, 092015 (2012).

276. CDF Collaboration (A. Abulencia et al.), Phys. Rev. D 75, 112001 (2007).

277. CDF Collaboration (T. Aaltonen et al.), Phys. Rev. D 82, 052005 (2010).

278. CDF Collaboration (T. Aaltonen et al.), Phys. Rev. D 80, 052003 (2009).

279. CDF Collaboration (T. Aaltonen et al.), Phys. Rev. Lett. 105, 131801 (2010).

280. CDF Collaboration (T. Aaltonen et al.), Phys. Rev. D 85, 011104 (2012).

281. CDF Collaboration (T. Aaltonen et al.), Phys. Rev. Lett. 107, 042001 (2011). 